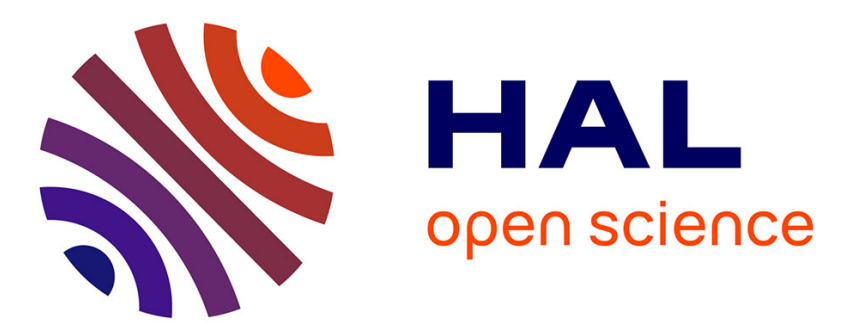

\title{
Modeling the respiratory chain complexes with biothermokinetic equations - The case of complex I.
}

\author{
Margit Heiske, Christine Nazaret, Jean-Pierre Mazat
}

\section{To cite this version:}

Margit Heiske, Christine Nazaret, Jean-Pierre Mazat. Modeling the respiratory chain complexes with biothermokinetic equations - The case of complex I.. BBA - Biochimica et Biophysica Acta, 2014, 1837 (10), pp.1707-1716. 10.1016/j.bbabio.2014.07.013 . hal-01058822

\section{HAL Id: hal-01058822 \\ https://hal.science/hal-01058822}

Submitted on 28 Aug 2014

HAL is a multi-disciplinary open access archive for the deposit and dissemination of scientific research documents, whether they are published or not. The documents may come from teaching and research institutions in France or abroad, or from public or private research centers.
L'archive ouverte pluridisciplinaire HAL, est destinée au dépôt et à la diffusion de documents scientifiques de niveau recherche, publiés ou non, émanant des établissements d'enseignement et de recherche français ou étrangers, des laboratoires publics ou privés. 


\section{Elsevier Editorial System(tm) for BBA - Bioenergetics}

Manuscript Draft

Manuscript Number: BBABIO-14-3R2

Title: Modelling the respiratory chain complexes with biothermokinetic equations - The case of complex I

Article Type: Regular Paper

Keywords: Complex I; rate equations; OxPhos Model

Corresponding Author: Prof. Jean Pierre Mazat,

Corresponding Author's Institution: Université Bordeaux 2

First Author: Margit HEISKE

Order of Authors: Margit HEISKE; Christine NAZARET; Jean Pierre Mazat

Abstract: The mitochondrial respiratory chain plays a crucial role in energy metabolism and its dysfunction is implicated in a wide range of human diseases. In order to understand the global expression of local mutations in the rate of oxygen consumption or in the production of ATP it is useful to have a mathematical model in which the changes in a given respiratory complex are properly modeled.

Our aim in this paper is to provide thermodynamics respecting and structurally simple equations to represent the kinetics of each isolated complexes which can, assembled in a dynamical system, also simulate the behaviour of the respiratory chain, as a whole, under a large set of different physiological and pathological conditions. On the example of the NADH-ubiquinol-oxidoreductase (complex I) we analyse the suitability of different types of rate equations. Based on our kinetic experiments we show that very simple rate laws, as those often used in many respiratory chain models, fail to describe the kinetic behaviour when applied to a wide concentration range. This led us to adapt rate equations containing the essential parameters of enzyme kinetic, maximal velocities and Henri-Michaelis-Menten like-constants (KM and KI) to satisfactory simulate these data.

Response to Reviewers: I will ask you to consider the argument of Reviewer \#3, requesting some changes in text and some text deletion (e.g., concerning Figure 4, which now does not appear). Please amend the manuscript accordingly.

Response :

- $\quad$ The discussion of MAL and NET equations was markedly reduced in Results section and moved to supplementary materials section S1. The MAL and NET equations were removed from table 1.

- $\quad$ The introduction of the proton gradient in the rate equation was no longer approached in the paper and the corresponding parts were suppressed in the highlights, the abstract, the results (section III) and the discussion. The previous Fig.4 was already suppressed in the first revised version. 


\section{$\frac{v u k}{3}$ \\ UNIVERSITÉ BORDEAUX

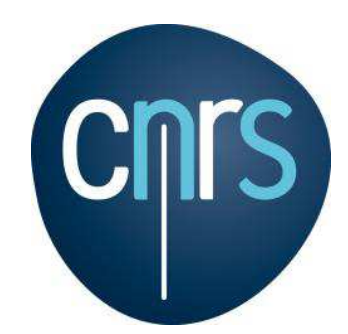

\section{Laboratoire de métabolisme énergétique cellulaire}

Bordeaux the $9^{\text {th }}$ of July 2014

Manuscript No.: BBABIO-14-3

"Modelling the respiratory chain complexes with biothermokinetic equations - The case of complex I" (Margit Heiske, Christine Nazaret, Jean-Pierre Mazat)

Dear editor-in-chief,

We followed the recommendations of reviewer \#3:

- The discussion of MAL and NET equations was markedly reduced in Results section and moved to supplementary materials section S1. The MAL and NET equations were removed from table 1.

- The introduction of the proton gradient in the rate equation was no longer approached in the paper and the corresponding parts were suppressed in the highlights, the abstract, the results (section III) and the discussion. The previous Fig.4 was already suppressed in the first revised version.

We really hope that in its new form our revised paper will be acceptable for publication in BBA Bioenergetics.

Yours sincerely

Prof. Jean-Pierre MAZAT 


\section{Response to Reviewers}

I will ask you to consider the argument of Reviewer \#3, requesting some changes in text and some text deletion (e.g., concerning Figure 4, which now does not appear). Please amend the manuscript accordingly.

Response :

The discussion of MAL and NET equations was markedly reduced in Results section and moved to supplementary materials section S1. The MAL and NET equations were removed from table 1.

The introduction of the proton gradient in the rate equation was no longer approached in the paper and the corresponding parts were suppressed in the highlights, the abstract, the results (section III) and the discussion. The previous Fig.4 was already suppressed in the first revised version. 
Graphical Abstract (for review)
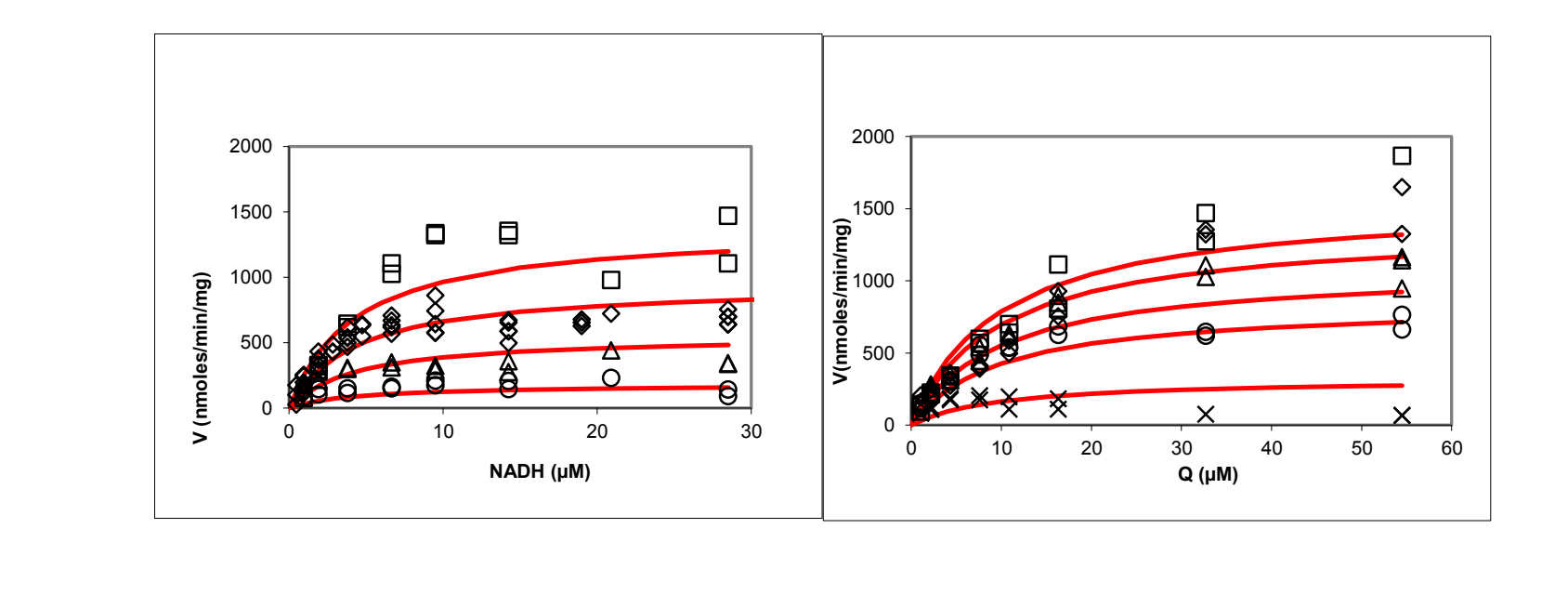

\section{Graphical Abstract (for review)}
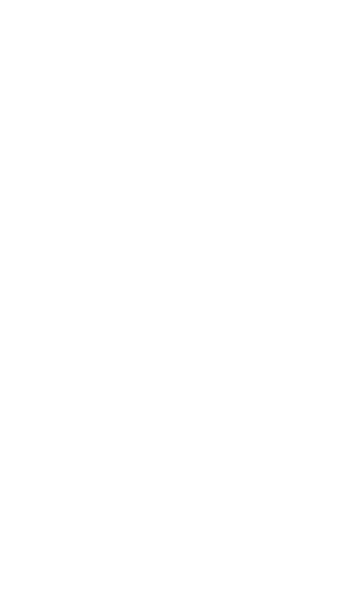
Highlights (for review)

- Necessity of a simple and realistic rate equation of the respiratory complexes.

- Near Equilibrium Thermodynamics rate equation and Mass Action Law are inadequate

- Extended-Reversible Henri-Michaelis-Menten (ER-HMM) equation is appropriate. 


\title{
Modelling the respiratory chain complexes with biothermokinetic equations - The case of complex I
}

\author{
Margit Heiske ${ }^{1,2,4}$, Christine Nazaret $^{3}$, Jean-Pierre Mazat ${ }^{1}$
}

${ }^{1}$ Université de Bordeaux, Bordeaux, France et Laboratoire de métabolisme énergétique cellulaire, IBGC - CNRS UMR 5095, 1 Rue Camille Saint Saëns, 33077 Bordeaux France.

${ }^{2}$ Institut für Biologie Theoretische Biophysik Humboldt-Universität zu Berlin, Invalidenstraße 42, Berlin,

${ }^{3}$ Institut de Mathématiques de Bordeaux, ENSTBB-Institut Polytechnique de Bordeaux

${ }^{4}$ Present address : Laboratoire d'Anthropologie Moléculaire et Imagerie de Synthèse, AMIS - CNRS UMR 5288, Université Paul Sabatier Toulouse III, 37 Allées Jules Guesde, 31073 Toulouse cedex

Keywords : Complex I, Oxidative phosphorylation, Modelling,

Highlights :

- Necessity of a simple and realistic rate equation of the respiratory complexes.

- Near Equilibrium Thermodynamics rate equation and Mass Action Law are inadequate

- Extended-Reversible Henri-Michaelis-Menten (ER-HMM) equation is appropriate.

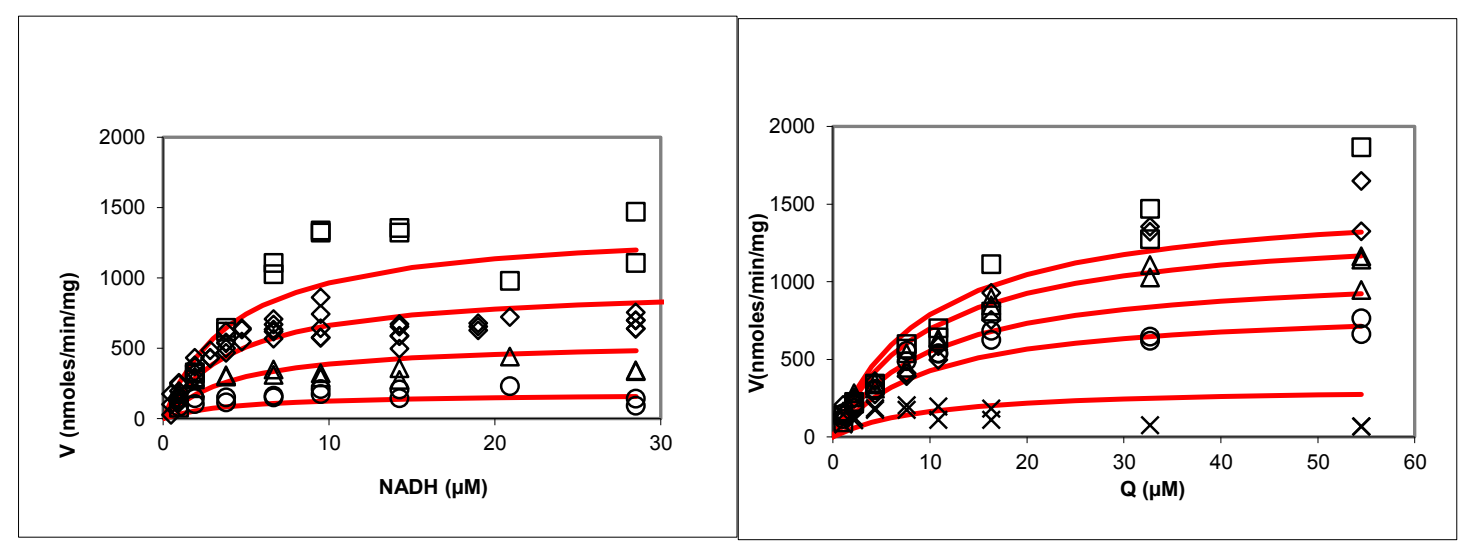




\begin{abstract}
The mitochondrial respiratory chain plays a crucial role in energy metabolism and its dysfunction is implicated in a wide range of human diseases. In order to understand the global expression of local mutations in the rate of oxygen consumption or in the production of ATP it is useful to have a mathematical model in which the changes in a given respiratory complex are properly modeled.

Our aim in this paper is to provide thermodynamics respecting and structurally simple equations to represent the kinetics of each isolated complexes which can, assembled in a dynamical system, also simulate the behaviour of the respiratory chain, as a whole, under a large set of different physiological and pathological conditions. On the example of the NADH-ubiquinol-oxidoreductase (complex I) we analyse the suitability of different types of rate equations. Based on our kinetic experiments we show that very simple rate laws, as those often used in many respiratory chain models, fail to describe the kinetic behaviour when applied to a wide concentration range. This led us to adapt rate equations containing the essential parameters of enzyme kinetic, maximal velocities and Henri-Michaelis-Menten likeconstants $\left(\mathrm{K}_{\mathrm{M}}\right.$ and $\left.\mathrm{K}_{\mathrm{I}}\right)$ to satisfactory simulate these data.
\end{abstract}




\section{Introduction}

The respiratory chain plays a crucial role in energy metabolism. In many cases, it consists of four enzyme complexes which are connected through two electron transporters, ubiquinone and cytochrome $c$. Three of the respiratory complexes extrude protons from the mitochondrial matrix into the intermembrane space liberating, step by step, the energy of the transfer of electrons from the low redox potential of the substrates $\mathrm{NADH}$ or $\mathrm{FADH}_{2}$ to the high redox potential of oxygen, energy then used to synthetize ATP.

It is now well documented that respiratory chain dysfunction is responsible for a wide range of human diseases including neurodegenerative diseases and cancer. Respiratory chain dysfunction may also have a possible relationship with aging [1-2] and metabolic disorders [3]. In many cases respiratory chain dysfunctions are due to mutations in the subunit constituting the respiratory chain complexes.

In order to better understand the behaviour of the respiratory chain in different physiological conditions and how the effects of pathological mutations are expressed at the global level of oxygen consumption or ATP synthesis it is useful to have a theoretical model of the respiratory chain. In other words understanding the normal or pathological interplay between these complexes and the electron transporters in the global functioning of the respiratory chain requires a model of each respiratory complex with a specific rate equation and then the integration of all the rate equations into a dynamical system representing the operation of the whole oxidative phosphorylation.

Several models, with different levels of complexity, have been established to describe the respiratory chain or its isolated complexes. It does not enter the scope of this article to review them all. We would just like to analyse the way in which the behaviour of each individual respiratory complex is approached in these models of the whole respiratory chain. It means that we discard of our analysis all the models in which the respiratory chain is represented by only one (or two depending on the electron entry point) equations (typically Magnus and Keizer [4], Cortassa et al. [5] and many others). Among the remaining models of respiratory chain two types of modelling of the individual complexes are used. The first significant models were developed in the framework of Non-Equilibrium Thermodynamic model (NET) involving a linear dependence of the flux as a function of the thermodynamic forces [6-9]. The model developed by Korzeniewski and Froncisz (1991) [10,11], is a comprehensive model also well representative of this class of thermodynamics models, extended thereafter ([12], [13]). 
Another approach, largely used to model the isolated respiratory complexes is the Mass Action Law (MAL), with the forward rate proportional to the product of substrats and the backward proportional to the product of products of the reaction. Bohnensack (1981) [14] was probably the first to derive a quantitative model of the respiratory chain involving nearly all the components of oxidative phosphorylation. In this approach the respiratory complexes were modeled according to the mass-action law. A recent example of this approach was proposed by Beard [15].

The advantage of both approaches (NET and MAL) is that they use very simple rate laws for each of the complexes with only one parameter to adjust. But these equations cannot reflect the respiratory complexes behaviours over a wide range of physiological conditions as it will be shown later in this paper.

As a matter of fact, the behaviour of the individual complexes is rather complex for several reasons: (i) the mechanisms catalysed by each separate complex are complex in themselves. The most illustrating example is the Q-cycle operating in complex III with two different quinone binding sites and the bifurcation of the electrons coming from $\mathrm{QH} 2$ molecule into two pathways. The case of cytochrome $c$ oxidase (complex IV) also involves a complex synchronisation between the 4 electrons necessary to reduce oxygen in water and the protons pumping. The case of complex I is not yet entirely resolved but already includes a cascade of 7 or 8 elementary redox reactions. (ii) not only substrates, products and modifiers play a role in the kinetics but also the electrochemical proton gradient which has an important influence on the activity of each complex and thus on the whole respiratory chain. (iii) the regulation of the activity of these complexes depends on allosteric conformations or on chemical modifications such as phosphorylation and acetylation [16-19].

On the one hand, the derivation of equations describing very precisely the underlying mechanisms of the respiratory complexes often involves a huge number of parameters which are hard or impossible to determine experimentally and will be in any case underdetermined [20-21]. On the other hand, the experimental kinetics of these complexes show, most of the time, the traditional pattern of enzymatic kinetics i.e. saturation behaviour. Furthermore, the recorded kinetics have been analysed with the usual phenomenological approaches of enzymology, particularly with the determination of $V_{\max }, K_{M}$ and $K_{I}$ at least for some of the substrates or products. It is well illustrated in the measurement of the (maximal) activity of the individual complexes, which is particularly useful in the diagnosis of mitochondrial diseases in attributing at least in some cases the pathology to a particular defect in a given complex as in [22]. In the same time the activity of the whole respiratory chain (and of part of 
TCA cycle) is recorded classically in very different conditions (resting state with a maximal transmembrane proton potential (state 4), phosphorylating state with a lower proton potential (state 3), intermediate states between states 4 and 3, uncoupled state etc.). In all these physiological conditions, the concentrations of the substrates/products of the individual respiratory complexes (NAD/NADH, Q/QH2, redox state of Cyt $c$ ) are also very different and presumably also different from the concentrations used in the determination of the (maximal) activity of the individual complexes. This could invalidate the use of the parameters values determined in these latter conditions (maximal activity determination in the absence of products) for the modelling of the whole respiratory chain functioning at non maximal activity in the presence of products.

For this reason we studied the kinetics of the isolated respiratory complexes in a broad range of substrates/products concentrations with the first aim to integrate the observations in a rate equation as simple as possible but nevertheless illustrating the main characteristics of the kinetics, particularly the saturation behaviour, the presence of products and the thermodynamic constraints. Our aim is to use these "simple" rate equations both to model kinetics of the individual isolated complexes and also the global behaviour of the whole respiratory chain when all the respiratory complexes are considered together. We will analyze different types of equations already used (NET, MAL) and we will propose other equations [23-27], more able to fit the kinetic of respiratory complexes particularly when involved in the electron transport and protons translocation through the whole respiratory chain: Extended Reversible Henri-Michaelis Menten Equation (ER-HMM), Ping Pong Mechanism (PPM), ordered mechanism (OM), "Convenience Kinetics" (CK) and a new type of equation : Extended Mass Action (EMA) (table 1 and Fig. 1). As an example, we will compare all these equations in their ability to model our kinetic results already published in [28] on beef heart complex I (NADH-Coenzyme Q reductase activity). It catalyses the reaction:

$$
\mathrm{NADH}+\mathrm{H}^{+}+\mathrm{Q} \stackrel{=}{=} \mathrm{NAD}^{+}+\mathrm{QH}_{2}
$$

\section{Material and Methods}

\subsection{Preparation of mitochondria}

Frozen isolated beef heart mitochondria were a gift from Dr. Joel Lunardi, prepared according to [29]. In this preparation the final mitochondrial pellets were homogenized at a concentration of $40 \mathrm{mg} / \mathrm{mL}$ and the mitochondrial suspension was frozen in liquid nitrogen as small beads of $50 \mu \mathrm{L}$ volume and stored at $-80^{\circ} \mathrm{C}$. In our experiments, one bead was thawed 
and diluted (1/5), aliquoted and the aliquots are frozen again. Then for each experiment an aliquot is thawed and diluted once more (1/20) in the slightly hypo-osmotic reaction buffer in order to ensure that the membranes are broken and their potential is null. For the assay itself, the mitochondrial suspension was diluted again (final concentration: $4.4 \mu \mathrm{g}$ mitochondrial proteins in $1 \mathrm{ml})$. All assays were performed in temperature-controlled single wavelength spectrophotometer.

\subsection{Complex I (NADH-ubiquinone oxidoreductase) assay}

The assay was performed at $37^{\circ} \mathrm{C}$ according to [22] by following the decrease in absorbance at $340 \mathrm{~nm}$ resulting from the oxidation of $\mathrm{NADH}$ in $1 \mathrm{~mL}$ of medium containing $65 \mathrm{mM}$ KH2PO4 (pH 7.5), 2 mg BSA, 2 mM EDTA, $46 \mu \mathrm{M}$ antimycin A, $4.4 \mu \mathrm{g}$ mitochondrial protein and in control assays additionally $25 \mu \mathrm{M}$ rotenone. A constant ethanol concentration of $8 \mu \mathrm{L} / 1000 \mu \mathrm{L}$ was present in all assays. It means that the complement to this quantity was added when not brought by the added constituents. The concentrations of the substrates NADH and Q (decylubiquinone) are varied, as well as the concentrations of products NAD+ and $\mathrm{QH} 2$. The reaction was initiated by NADH addition. The extinction coefficient used for NADH concentration determination was $6.22 \mathrm{mM}^{-1} \mathrm{~cm}^{-1}$ at $340 \mathrm{~nm}$. The net activities have been obtained by subtracting the residual activity in the presence of rotenone from the activities without.

\subsection{Parameter estimations}

The parameters values of the different rate equations have been estimated minimizing the root mean square deviation (RMSD) between the experimental and theoretical data points. RMSD for one set of parameters was calculated simultaneously with all experimental series (see figures in the supplementary materials). To find the RMSD minimum, we used a global search routine (genetic algorithm) followed by a local one (quasi Newton based) as proposed in [30]. It permits to browse the whole parameter space and then to converge towards a well defined solution, usually unique in our cases. All calculations have been done using Scilab (Scilab Enterprises, 2012). Because the reaction catalyzed by the respiratory chain complex I is highly exergonic, the rate constant of the backward reaction $\mathrm{k}_{\mathrm{b}}$ is simply calculated via the Haldane relationship:

$K_{e q}=\frac{k_{f}}{k_{b}} \cdot \frac{K_{N A D}}{K_{N A D H}} \cdot \frac{K_{Q H_{2}}}{K_{Q}}$ (2) or equation $K_{e q}=\frac{k_{f}}{k_{b}} \frac{C_{P}}{C_{S}}$ (3) for EMA with the equilibrium constant: 


$$
K_{e q}=\exp \left(-\frac{\Delta G_{o}^{\prime}}{R T}\right)=1.54 \times 10^{+11}(4)
$$

where $\mathrm{R}=8.314 \mathrm{~J} / \mathrm{mol} / \mathrm{K}$. The temperature in the experiments was $\mathrm{T}=310 \mathrm{~K}$ and $\Delta \mathrm{G}^{\prime} \mathrm{o}=-$ $66.4 \mathrm{~kJ} / \mathrm{mol}$ at $\mathrm{pH} 7.5(69.4$ at $\mathrm{pH} 7.0)$.

\section{RESULTS}

\section{Non-saturable rate equations}

\section{I-1 Near Equilibrium Thermodynamics (NET) Equation}

In the near equilibrium approach the reaction velocity is assumed to be proportional to the Gibbs energy.

$v_{C I}=-k_{C I} \Delta G_{C I}$

with :

$$
\Delta G_{C I}=\Delta G_{o, C I}^{\prime}+R T \cdot \operatorname{Ln}\left[\frac{[N A D] \cdot\left[Q H_{2}\right]}{[N A D H] \cdot[Q]}\right]
$$

This type of equation was proposed by Westerhoff [7] and used by Korzeniewski et al. [10] for instance to model complexes I and III of respiratory chain.

\section{I-2 The Mass Action Law equation.}

The MAL equation applied to the enzymatic reaction (1) reads:

$v_{C I}=k_{f}[N A D H] \cdot[Q]-k_{b}[N A D] \cdot\left[Q H_{2}\right]$

or introducing $\mathrm{K}_{\mathrm{eq}}: v_{C I}=k_{b}\left(K_{e q}[N A D H] .[Q]-[N A D] .\left[Q H_{2}\right]\right)$

This type of equation was largely used for modelling the respiratory chain complexes (e.g. [14-15]).

We conclude that these equations (see supplementary materials S1), MAL as well as the NET equation cannot be used for an accurate description of respiratory complexes kinetics when there is a possible variation in substrates and products concentrations. It is necessary to introduce a saturation term in the rate equations.

II The saturable rate equations.

\section{II-1 The equations}


The list of the rate equations tested in this work which exhibit a saturable pattern as a function of substrate or product concentrations are listed in table 1 and their mechanism depicted in Fig. 1. They all involve the same type of numerator as MAL equation vanishing at equilibrium (or an equivalent expression in the case of EMA). In addition, they also involve a denominator with Michaelis constants $\mathrm{K}_{\mathrm{M}}$ for substrates and products. Because we look for an equation as simple as possible we assume that the $\mathrm{K}_{\mathrm{M}}$ for a substrate/product is independent of the binding of the others which is not necessarily true in the reality. Because the denominators of the equations are different, one can predict that the values of $\mathrm{K}_{\mathrm{M}}$ may be different for the different equations. All these equations but PPM are equivalent in absence of products.

\section{Table 1 and Fig. 1}

The Extended-Reversible Henri-Michaelis-Menten (ER-HMM) equation [23-25; 31-34] corresponds to a random binding/release of substrates/products as represented in Fig. 1. It is well described in all textbooks (see [26] for instance).

The convenience kinetics equation (CK) is a generic equation which can account for any number of substrates and products. It has been proposed by Liebermeister and Klipp [27] and includes the same basic kinetic parameters (linked by the same Haldane relationship) than ER-HMM equation above but arranged slightly differently in the denominator so that the Km values may be different. In the case of 2 substrates 2 products, it also corresponds to a random binding mechanism but, in contrast to ER-HMM mechanism, without the non-reactive ternary complexes E-NADH-QH2 and E-NAD-Q (see Fig. 1).

The interests of ER-HMM equation is well explained by Cornish-Bowden and Hofmyer in [23] which also applies to CK: minimal number of parameters, adherence to thermodynamic constraints, competition between substrates and their corresponding products and saturability.

The equation given in table 1 for the order mechanism (OM) correspond to the case NQNQ meaning that NADH binds first then $\mathrm{Q}$ and NAD is released first then $\mathrm{QH}_{2}$. The four possible sequences of substrates binding/products release have been tested (see table 2).

A ping-pong mechanism was also proposed for complex I kinetics (see the discussion in [28]). It can correspond to two situations: NNQQ or QQNN meaning that either NADH reacts first and gives $\mathrm{NAD}$ and then $\mathrm{Q}$ gives $\mathrm{QH}_{2}$ or vice versa. In the simplified form where the $\mathrm{K}_{\mathrm{M}}$ are independent of each other, both equations have the same expression given in table 1. 
Finally we tested EMA equation (Extended Mass Action) which is based on mass action according to two different possible mechanisms (see supplementary materials S7 for a complete derivation of this equation). It can also be seen as a simplified ER-HMM equation in which the Michaelis constants of the substrates (resp. the products) are fused in one constant $\mathrm{C}_{\mathrm{S}}\left(\right.$ resp. $\mathrm{C}_{\mathrm{P}}$ ). The equation has only 4 parameters which are not independent; one can be replaced by the equilibrium constant via the Haldane-like relationship:

$K_{e q}=\frac{k_{f}}{k_{b}} \frac{C_{P}}{C_{S}}$

This equation has the big advantage that any stoichiometric factor (also non integer) can be included, like for the NET and MAL equations. This equation was developed independently by Liebermeister et al. [35].

For each equation, the results of parameters fitting using all the experimental points are listed in table 2. Fig. 2e to $\mathrm{h}$ gives an example of these fittings in the case of ER-HMM and EMA equation with only some of the experimental points. The figures showing the results of the fittings according to other saturable rate laws with the complete set of experimental points are shown in supplementary materials. The results are always plotted in comparison with the ERHMM fit (blue dashed).

\section{Table 2 and Fig. 2}

Comparing the RMSD (table 2), it is clear that all equations but EMA give nearly the same good accuracy. The rate constants and the substrates $\mathrm{K}_{\mathrm{M}}$ values are also very similar (except for EMA for which the parameters have different meanings). An average value of $\mathrm{k}_{\mathrm{f}}=1810 \pm$ 43 nmoles. $\min ^{-1} \cdot$ prot $^{-1}, \mathrm{~K}_{\mathrm{NADH}}=4.6 \pm 0.7 \mu \mathrm{M}$ and $\mathrm{K}_{\mathrm{Q}}=10.2 \pm 1.3 \mu \mathrm{M}$ are obtained. This is not the case for the $K_{M}$ values of the products. The determination of the products $K_{M}$ is more difficult because as mentioned above, the complex I reaction is largely irreversible in the absence of $\Delta \mu_{\mathrm{H}}{ }^{+}$. The only way to have an indication of the products $\mathrm{K}_{\mathrm{M}}$ is to record the reaction rate in the forward direction in the presence of different concentrations of the products (Fig. 3). The values of the products $\mathrm{Km}$ are dependent on the structure of the denominator of each equation, but still one can state that $\mathrm{K}_{\mathrm{NAD}}$ is clearly high and difficult to measure indicating a low affinity of $\mathrm{NAD}^{+}$. On the other hand $\mathrm{K}_{\mathrm{QH} 2}$ is rather low, sometimes lower than $\mathrm{K}_{\mathrm{Q}}$. Both $\mathrm{K}_{\mathrm{m}}$ products depend upon the rate equation used to fit the experimental results.

\section{Figure 3 a et $b$}




\section{II-2 Q inhibition}

Figure 4 shows a clear inhibition at high Q concentration (> $100 \mu \mathrm{M})$. Different type of substrate inhibitions can be considered such as non-competitive, substrate/steric inhibition (at the normal binding site). On the example of ER-HMM equation, several inhibition terms have been tested. The ER-HMM equation reads then:

$v=E_{t} I_{n c} \frac{k_{f} \cdot \frac{N A D H}{K_{N A D H}} \cdot \frac{Q}{K_{Q}}-k_{b} \cdot \frac{N A D}{K_{N A D}} \cdot \frac{Q H_{2}}{K_{Q H_{2}}}}{\left(1+\frac{N A D H}{K_{N A D H}}+\frac{N A D}{K_{N A D}}\right)\left(1+\frac{Q}{K_{Q}} I_{S}+\frac{Q H_{2}}{K_{Q H_{2}}} I_{S}\right)}$

with the inhibition terms $I_{s}$ and $I_{n c}$ described in the following.

\section{Substrate-product or steric inhibition $I_{S}$}

This type of inhibition corresponds to the obstruction of the catalytic site of quinone reduction by quinone or quinol. Assuming that $\mathrm{Q}$ and $\mathrm{QH}_{2}$ obstruction has the same effect on the binding of both molecules $\mathrm{QH}_{2}$ or $\mathrm{Q}$, the term $\mathrm{I}_{\mathrm{s}}$ can be written:

$I_{S}=1+\frac{[Q]}{K_{i 1}}+\frac{\left[Q H_{2}\right]}{K_{i 2}}$

where $\mathrm{K}_{\mathrm{i} 1}$ and $\mathrm{K}_{\mathrm{i} 2}$ are the inhibition constants of $\mathrm{Q}$ and $\mathrm{QH}_{2}$ respectively. In the case where $\mathrm{K}_{\mathrm{i} 1}=\mathrm{K}_{\mathrm{i} 2}=\mathrm{K}_{\mathrm{i}}$, the expression (10) becomes:

$I_{S}=1+\frac{\left[Q_{t o t}\right]}{K_{i}}$ where $\left[\mathrm{Q}_{\mathrm{tot}}\right]=[\mathrm{Q}]+\left[\mathrm{QH}_{2}\right]$

\section{Non competitive inhibition $I_{n c}$}

Another possibility is that $\mathrm{Q}$ or/and $\mathrm{QH}_{2}$ bind to a second quinone site on the molecule, modifying the activity of complex I, giving rise to a non-competitive inhibition.

The generic expression of a non-competitive inhibition is:

$I_{n c}=\left(\frac{1}{1+\frac{[Q]}{K_{i 1}}+\frac{\left[Q H_{2}\right]}{K_{i 2}}}\right)^{n}$ 
The values $n=1$ and $n=2$ have been tested with inhibition by $Q_{\text {tot }}\left(\right.$ with $K_{i 1}=K_{i 2}=K_{i}$ ) or $Q$ alone.

All the experimental points have been fitted by ER-HMM equation (9) involving different $I_{s}$ or $\mathrm{I}_{\mathrm{nc}}$ terms listed in table 3 (see supplementary materials Fig. S6 and S7).

Very similar accuracies of the different fits are obtained. In the case of steric inhibition, taking $\mathrm{Q}$ and $\mathrm{QH}_{2}$ separately or $\mathrm{Q}_{\text {tot }}$ as inhibitor makes no real difference (not shown). It is the same in the case of non-competitive inhibition. In this latter case taking $n=1$ or $n=2$ gives similar fits. The steric (red broad line) and non-competitive (black thin line) inhibitions are represented on figure 4 and overlap. The curve without inhibition (blue dashed line) is also shown for comparison. Interestingly the values of $\mathrm{K}_{\mathrm{Q}}$ (and of $\mathrm{K}_{\mathrm{NADH}}$ ) remain approximately the same for all types of inhibition. Only $\mathrm{K}_{\mathrm{QH} 2}$ changes significantly when part of the normal (product) $\mathrm{QH}_{2}$ inhibition is taken by the specific inhibitory mechanism.

\section{Table 3 and Fig. 4}

\section{DISCUSSION AND CONCLUSION}

\section{NET and MAL rate equations}

As obvious in Fig. 2 (a to d), Near Equilibrium Thermodynamics (NET) and Mass Action Law (MAL) rate equations fail to adequately represent the activity of complex I when the concentrations of substrates are varied. In the case of NET, the logarithmic term dampens the variations of substrates concentrations so that the rate value is nearly constant (between 540 and $650 \mathrm{nmol} / \mathrm{min} / \mathrm{mg}$ prot) over the nearly two order of magnitude of substrates concentrations used. Only at very low concentration of one substrate, the rate decreases rapidly toward zero (In fact to minus infinity; see the red line along the y-axis in Fig.2 a and b). It is not surprising because in conditions of very low products concentrations $(0.1 \mu \mathrm{M}$ each in our case to make the calculation possible) with a value of $\Delta \mathrm{G}^{\prime}{ }_{\mathrm{o}}=-66.4 \mathrm{~kJ}$, the reaction is far from equilibrium and its rate will not obeys the conditions of near equilibrium thermodynamics.

A slightly better fit is obtained with MAL equation, because in this case the rate equation is sensitive to substrates concentrations variations. However the linear increase of the rate as a function of substrates concentrations does not correspond to the reality of saturable kinetics. For comparison the fit of the same points with a typical saturable kinetic equation (ER-HMM, Fig. 2 e-f) is shown. However NET and MAL equations may be a good choice for the 
description of the respiratory chain in conditions close to equilibrium which can be the case for complex I, II and III of respiratory chain in in vivo conditions with $\Delta \mu_{\mathrm{H}^{+}} \neq 0$. It must be stressed that in all cases, the constants involved in these equations $\left(\mathrm{k}_{\mathrm{CI}}\right.$ or $\left.\mathrm{k}_{\mathrm{f}}\right)$ get ad hoc values which do not correspond to any intrinsic property (kinetic constants) of the respiratory complexes.Nevertheless, as demonstrated by Pillay et al. [36], a MAL model can give rise to a "saturation" behaviour particularly in redox cycles when the sum of the redox couples (NAD/NADH and Q/QH2) is constant. In these conditions, the maximal velocity depends upon the rate constant and the total concentrations of substrates/products and the half saturation concentration also depends upon the total concentration of the redox couples and is thus variable and different of a real $\mathrm{Km}$. Using such equations will make it difficult to analyze the effect on OXPHOS of a variation of a particular kinetic parameter ( $\mathrm{k}_{\mathrm{cat}}$ or $\mathrm{Km}$ ) of a given respiratory complex or of physiological or pathological changes in total substrate and product concentrations.

On the contrary Fig. 2 e-h (and Fig. S2 to S7 in supplementary materials) and table 2 show that all the other equations tested in our study give similar good fits when compared with the whole set of our experimental data. It should be however noticed that EMA fit, which involves two parameters less, is less accurate mainly because it does not have independent saturation terms for each of the substrates/products. Their binding is rather considered simultaneous (see Supplementary Materials) and quantified by the phenomenological constants Cs and Cp. When the Km of substrates (respectively the products) are similar the phenomenological constants $\mathrm{C}$ represent adequately their association by an average constant. It is no longer the case when the Km are different as here for NAD and NADH.

\section{Kinetic mechanism and kinetic constants}

The striking results of this study that several kinetic equations are equally able to fit the experimental results over large concentrations of substrates and products is consistent with the fact that the kinetic mechanism of this reaction is still a matter of discussion. It means that this form of analysis does not shed light on the, too complex, kinetic mechanism of the reaction. Fato et al. [37] proposed a ping-pong mechanism in the case of mitochondria isolated from bovine heart with the oxidation of NADH preceding the reduction of ubiquinone. Nakashima et al. [38] used $\mathrm{CoQ}_{1}$ as the electron acceptor to analyze the activity of complex I purified from bovine heart. They proposed an ordered sequential mechanism with $\mathrm{CoQ}_{1}$ binding as the first step and $\mathrm{CoQ}_{1} \mathrm{H}_{2}$ releasing as the last step. Hano et al. [39] assumed that the kinetics of 
complex I obeys an ordered sequential mechanism when they used decylubiquinone (DQ) as the electron acceptor.

Analyzing the same set of experimental results as in this study in the light of a stochastic model based on Gillespie approach [40] and taking into account the structure (distances) and the midpoint potentials of the reaction centers we showed that the kinetics may not necessarily obey a simple mechanism (ordered or ping-pong) [28]. This is particularly due to the substantial distance (around $90 \AA$ ) between NADH oxidation site and quinone reduction site and the presence of seven redox reactions in between. It makes the two extreme redox sites (NADH/NAD on the one hand and Q/QH2 on the other hand) as if they were independent from each other. The stochastic simulations also evidenced a plateau for saturating NADH concentrations (see supplementary figures a-j). It renders the fit by any equation used in this study slightly inaccurate: at high NADH concentrations the theoretical curves go on increasing weakly, while the experimental rates (as well as the stochastic simulations) are steady and quasi horizontal. We showed that this does not result from a substrate inhibition but simply from the accumulation of electrons in the intermediate redox centers as NADH concentration increases, leading to a sort of electron buffering effect [28]. This can be easily understood in the following way: if the rate of the second half of the mechanism $(\mathrm{Q}=>\mathrm{QH} 2)$ is low as compared to first one (NADH $=>\mathrm{NAD})$ and to the intermediate redox reactions inside complex I, for instance low Q and high NADH, then the electrons accumulate progressively backwards on the 7-8 FeS centers in between the two half reactions. As soon as one QH2 molecule is formed, two electrons from some FeS centers are again rapidly available. The limiting step is the release of the previously formed QH2 molecule and the binding of the new Q molecule which are phenomena much slower than the "flitting" of the electrons between the FeS centers and operating at constant rate when Q and $\mathrm{QH}_{2}$ are constants. In other words, when the second half reaction is slower than the first one, there are always two electrons to reduce a $\mathrm{Q}$ molecule when present. It is the reason why a plateau is observed in the kinetics particularly at high NADH and low Q.

Another salient feature of our study is that for all the saturable equations (but EMA) the maximal rate constants and the $\mathrm{Km}$ are nearly the same (see table 2) and well comparable with the data in the literature on beef heart mitochondria complex I. For instance we find a forward maximal rate constant between 1773 and $1910 \mathrm{nmol} \mathrm{NADH} . \mathrm{min}^{-1} \cdot \mathrm{mg}^{-1}$. Fato et al. [37] determined on submitochondrial particles for different types of quinone a $\mathrm{k}_{\mathrm{cat}}$ ranging from 170 to $1560 \mathrm{nmol}$ NADH. $\mathrm{min}^{-1} \cdot \mathrm{mg}^{-1}$. Sherwood and Hirst [41] reported a value of 3 $100 \mathrm{nmol} \mathrm{NADH} \cdot \mathrm{min}^{-1} \cdot \mathrm{mg}^{-1}$ and Hano et al. [39 ] a value of $1860 \mathrm{nmol} \mathrm{NADH} \cdot \mathrm{min}^{-1} \cdot \mathrm{mg}^{-1}$. 
Lower values around $500 \mathrm{nmol} \mathrm{NADH} . \mathrm{min}^{-1} \cdot \mathrm{mg}^{-1}$ were reported by other authors with different quinone analogs [42].

$\mathrm{K}_{\mathrm{m}}(\mathrm{NADH})$ was found in the range of $0.6-2.7 \mu \mathrm{M}$ on isolated complex I in the presence of different quinone types, among them also decylubiquinone [38, 39], always in beef heart mitochondria. For SMP a value of $9.2 \mu \mathrm{M}$ has been found [37]. Vinogradov [43] reported for $\mathrm{K}_{\mathrm{m}}(\mathrm{NADH}) 7.6 \mu \mathrm{M}$ and $7.2 \mu \mathrm{M}$ for coupled and uncoupled SMPs, respectively. Nakashima et al. [38] found a $\mathrm{K}_{\mathrm{m}}(\mathrm{NADH})$ around $2 \mu \mathrm{M}$ depending of Q1 and NAD concentrations. It is well comparable with the values $4.2-6.1 \mu \mathrm{M}$ we found in our fits.

The values found for $\mathrm{K}_{\mathrm{m}}(\mathrm{Q})$ are more variable, due to the different type of quinone that has been used. Fato et al. [37] determined the $K_{m}(Q)$ for 7 different types of quinones on bovine submitochondrial particles. Their values for decylubiquinone (DQ) were found to be 1.8 and $2.1 \mu \mathrm{M}$. A higher value of $24 \mu \mathrm{M}$ has also been reported in [41]. For complex I isolated from beef heart, values of $4.4-12.9 \mu \mathrm{M}$ for Q1 and Q2 $[38,39,44]$ have been reported and $51 \mu \mathrm{M}$ [39] for decylubiquinone. These values are similar to the values between 9.5 and 13.8 found in our fits.

Less data are available with respect to the Km values of the products and they are more difficult to compare to our values because of their dependence on the rate equations structures. Vinogradov [45] found a $\mathrm{K}_{\mathrm{i}}$ value for NAD of $1250 \mu \mathrm{M}$ on uncoupled SMP, which lies among the K-values we found for the different equations. However, for the reverse reaction under coupled conditions the author reported a $\mathrm{Km}$ of $7.2 \mu \mathrm{M}$ for NAD. To explain the difference of about three orders of magnitude it was suggested that NAD binds to a different site for the reverse sense. But this large difference may also be due to the equation that has been used for the determination of these values, as we can see on tables 2 and 3 . With only three independent parameters (instead of five for other equations ER-HMM, PPM or $\mathrm{OM}$ ), the EMA equation is a significantly less precise description of the given data. However, for most data curves EMA is still comparable to the other rate equations, as one can see in Fig. 2 and on Fig. S2 . But since the product inhibition by $\mathrm{QH}_{2}$ and $\mathrm{NAD}^{+}$are approximately three orders of magnitude apart, it is obvious that EMA cannot describe accurately the influence of both. Here the fits lead to a good description of the influence of $\mathrm{QH}_{2}$, but in contrast, in the series where $\mathrm{NAD}^{+}$was varied the data description is not satisfactory. For the latter no $\mathrm{QH}_{2}$ was present which means that the product term in the EMA equation was always 0 , with $\mathrm{NAD}^{+}$present or not. Hence EMA is not applicable to such extreme situation 


\section{Inhibition by $Q$.}

In our experimental data one can observe an inhibition of complex I at high concentrations of decylubiquinone (Fig. 4). Indeed oxidized quinones have been suspected to exert a negative effect on complex I activity at higher concentrations. Lenaz et al. [46] showed an inhibitory effect by the short chain CoQ analogue $\mathrm{Q}_{3}$ and assumed that there is need for long chain ubiquinone for a proper functioning of complex I. Other authors reported this inhibition with different types of quinones [37] and [44]. Grivennikova et al. [18] reported even a very strong inhibition by the short chain ubiquinone. This could suggest the existence of a second inhibitory site for quinone (oxidized) in addition for the substrate site as we already discussed in [28] (see also [41], [44], [47-50]. Althoug,there is no evidence of a precise second ubiquinone site, there is the possibility of ubiquinone taking several positions either in the large reaction pocket or on the way leading from the membrane to the reaction site and thus impeding or hindering the access of the ubiquinone substrate by ubiquinol or the release of the ubiquinol product by ubiquinone, well modeled by a steric inhibition either by Q or QH2. It should be noticed that the inhibitory concentration of $\mathrm{Q}$ are rather high $(>100 \mu \mathrm{M})$ and that $15-20 \%$ inhibition is obtained at $350 \mu \mathrm{M}$ Q. Thus the inhibition term can be presumably neglected in most of the cases.

\section{Choice of a rate equation}

Because all equations but EMA give a similar good fit of our kinetic experiments performed with large variations of the substrates and products, we select the ER-HMM equation to represent complex I activity. It is among the simplest and corresponds to the random bi-bi mechanism. As noted above, the inhibitory term is probably most of the time superfluous. If necessary, it will be added under the form of a non competitive inhibition by $\mathrm{Q}_{\text {tot }}$ (see Fig. S5 and S6). Both type of inhibition (non competitive and steric) give very comparable inhibitory pattern (Fig. S5 and S6).

$v=E_{t} I_{n c} \frac{k_{f} \cdot \frac{N A D H}{K_{N A D H}} \cdot \frac{Q}{K_{Q}}-k_{b} \cdot \frac{N A D}{K_{N A D}} \cdot \frac{Q H_{2}}{K_{Q H_{2}}}}{\left(1+\frac{N A D H}{K_{N A D H}}+\frac{N A D}{K_{N A D}}\right)\left(1+\frac{Q}{K_{Q}}+\frac{Q H_{2}}{K_{Q H_{2}}}\right)} \quad$ (13) with $I_{n c}=\left(\frac{1}{1+\frac{\left[Q_{t o t}\right]}{K_{i}}}\right)$

We have shown that this type of equation (ER-HMM) can be used to fit the kinetics of the other complexes of the respiratory chain [51]. 
In a forthcoming paper we will discuss the introduction of the proton gradient in biothermokinetic rate equations and analyze different approaches with respect to their ability to reproduce OXPHOS data under coupled conditions.

\section{ACKNOWLEDGEMENTS}

The authors are indebted to Drs. Joel Lunardi and Gaëlle Hardy for the preparation of mitochondria, to Dr. S. Ransac for fruitful discussions and to Prof. Edda Klipp for her constant support.

\section{REFERENCES}

[1] M., Frenzel, H. Rommelspacher, M. D. Sugawa, N. A. Dencher, Ageing alters the supramolecular architecture of OxPhos complexes in rat brain cortex. Exp. Gerontol. 45 (2010) 563-572.

[2] D. C. Wallace, Mitochondrial DNA mutations in disease and aging. Environ. Mol. Mutagen. 51 (2010) 440-450.

[3] S. DiMauro, E. A. Schon, Mitochondrial disorders in the nervous system. Annu. Rev. Neurosci. 31, (2008) 91-123.

[4] G. Magnus, J. Keizer.. Minimal model of b-cell mitochondrial Ca21 handling. Am. J. Physiol. 273. Cell Physiol. 42 (1997) C717-C733.

[5] S.Cortassa, M.A.Aon, E.Marban, R.L.Winslow, B. O'Rourke,. An integrated model of cardiac mitochondrial energy metabolism and calcium dynamics. Biophys J. 84 (2003) 273455 .

[6] H. Rottenberg, Non-equilibrium thermodynamics of energy conversion in bioenergetics, Biochim. Biophys. Acta 549(1979) 225-253.

[7] , H.V. Westerhoff, K. van Dam Thermodynamics and control of free-energy transduction, (1987) Elsevier, Amsterdam.

[8] J.W. Stucki,The thermodynamic-buffer enzymes. E. J. Biochem. 109 (1980) 257-267.

[9] J.W. Stucki, The optimal efficiency and the economic degrees of coupling of oxidative phosphorylation. E. J. Biochem. 109 (1980) 269-283.

[10] B. Korzeniewski, W. Froncisz, Theoretical studies on the control of the oxidative phosphorylation system, Biochim. Biophys. Acta 1102 (1992) 67-75.

[11] B. Korzeniewski, Regulation of ATP supply in mammalian skeletal muscle during resting state --> intensive work transition. Biophys Chem. 83 (2000)19-34. 
[12] B. Korzeniewski, J.-P. Mazat, Theoretical studies on the control of oxidative phosphorylation in muscle mitochondria at different energy demands and oxygen concentrations. Acta Biotheoretica 44 (1996) 263-269.

[13] B. Korzeniewski, M. Malgat, T. Letellier, J.-P. Mazat, Effect of binary mitochondria heteroplasmy on respiration and ATP synthesis: implications to mitochondrial diseases, Biochem. J. 357 (2001) 835-842.

[14] R. Bohnensack, Control of energy transformation of mitochondria. Analysis by a quantitative model, Biochim. Biophys. Acta 634 (1981) 203-218.

[15] D.A. Beard, A biophysical model of the mitochondrial respiratory system and oxidative phosphorylation. PloS Computational Biology 1 (2005) 252-264.

[16] S. Helling, M. Hüttemann, R. Ramzan, S. H. Kim, I. Lee, T. Müller, E. Langenfeld, H. E. Meyer, B. Kadenbach, S. Vogt, K. Marcus. Multiple Phosphorylations of Cytochrome $c$ Oxidase and their Functions. Proteomics. 12 (2012) 950-9.

[17] B. Kadenbach, R. Ramzana, L. Wenb, S. Vogt. New extension of the Mitchell Theory for oxidative phosphorylation in mitochondria of living organisms. Biochimica et Biophysica Acta 1800 (2010) 205-212.

[18] V. G. Grivennikova, A. N. Kapustin, A. D. Vinogradov. Catalytic activity of NADHubiquinone oxidoreductase (complex I) in intact mitochondria. Evidence for the slow active/inactive transition. J. Biol Chem, 276(2001) 9038-44,

[19] N. Capitanio, L.L. Palese, G. Capitanio, P.L. Martino, O.M. Richter, B. Ludwig, S. Papa, Allosteric interactions and proton conducting pathways in proton pumping aa(3) oxidases: heme a as a key coupling element. Biochim Biophys Acta 1817 (2012) 558-66.

[20] K.Yugi, M. Tomita, A general computational model of mitochondrial metabolism in a whole organelle scale. Bioinformatics 20 (2004) 1795-1796

[21] N.Berndt, S. Bulik, H.-G. Holzhütter, Kinetic Modeling of the Mitochondrial Energy Metabolism of Neuronal Cells: The Impact of Reduced $\alpha$-Ketoglutarate Dehydrogenase Activities on ATP Production and Generation of Reactive Oxygen Species. International Journal of Cell Biology Volume 2012 (2012), Article ID 757594, 11 pages doi:10.1155/2012/757594

[22] F. Medja, S. Allouche, P. Frachon, C. Jardel, M. Malgat, B. Mousson de Camaret, A. Slama, J. Lunardi, J.P. Mazat, A. Lombès, Development and implementation of standardized respiratory chain spectrophotometric assays for clinical diagnosis, Mitochondrion 9 (2009) 331-339. See also http://mitolab.eu/consensus-protocols-for-clinicaldiagnosis/

[23] A. Cornish-Bowden, J.-H.S. Hofmeyr, Enzyme in context: Kinetic characterization of enzymes for systems biology. The Biochemist, (April 2005) 11-14.

[24] C. Chassagnole, B. Raïs, E. Quentin, D.A. Fell, J.-P. Mazat, An integrated study of threonine pathway enzyme kinetics in Escherichia coli. Biochem. J. 356, (2001) 415-423. 
[25] J. M. Rohwer, Kinetic modelling of plant metabolic pathways Journal of Experimental Botany, 63 (2012) 2275-2292.

[26] I. H. Segel. Rapid equilibrium bireactant systems. In I. H. Segel, editor, Enzyme Kinetics. Behavior and Analysis of Rapid Equilibrium and Steady-State Enzyme Systems. John Wiley \& Sons, New York, wiley classics library edition, 1993. ISBN 978-0-471-30309-1.

[27] W. Liebermeister, E. Klipp, Bringing metabolic network to life: convenience rate law and thermodynamic constraints. Theor. Biol. and Med. Mod., 3 (2006) 41.

[28] S. Ransac, M. Heiske, J.-P. Mazat : From in silico to in spectro Kinetics of Respiratory Complex I. Biochim. Biophys. Acta 1817 (2012) 1958-1969.

[29] ] A.L. Smith, Preparation, properties, and conditions for assay of mitochondria: Slaughterhouse material, small-scale. Methods in Enzymology 10 (1967) 81-86.

[30] P. Mendes, D. Kell, Non-linear optimization of biochemical pathways: applications to metabolic engineering and parameter estimation, Bioinformatics 14 (1998) 869-883.

[31] V. Henri, Théorie générale de l'action de quelques diastases. Compte Rend. Acad. Sci. Paris 135 (1902) 916-919.

[32] L. Michaelis, M.L. Menten, Die Kinetic der Invertinwirkung. Biochem. Z. 49 (1913) 333-369.

[33] G.E. Briggs J.B.S. Haldane, A note on the kinetics of enzyme action. Biochem. J. 19 (1925) 338-339.

[34] U. Deichmann,S. Schuster,J.-P. Mazat,A. Cornish-Bowden, Commemorating the 1913 Michaelis-Menten paper Die Kinetik der Invertinwirkung: three perspectives. FEBS J. 281 (2014) 435-63. 
[35] W. Liebermeister, J. Uhlendorf, E. Klipp, Modular rate laws for enzymatic reactions: thermodynamics, elasticities and implementation Bioinformatics. 26 (2010) 1528-34.

[36] C.S. Pillay, J.-H. S. Hofmeyr, B.G. Olivier, J.L. Snoep, J.M. Rohwer, Enzymes or redox couples? The kinetics of thioredoxin and glutaredoxin reactions in a systems biology context. Biochem. J. 417 (2009) 269-275.

[37] R. Fato, E. Estornell, S. Di Bernardo, F. Pallotti, G. Parenti Castelli, G. Lenaz, Steady-state kinetics of the reduction of coenzyme $Q$ analogs by complex I (NADH:ubiquinone oxidoreductase) in bovine heart mitochondria and submitochondrial particles, Biochemistry 35 (1996) 2705-2716.

[38] Y. Nakashima, K. Shinzawa-Itoh, K. Watanabe, K. Naoki, N. Hano, S. Yoshikawa, Steadystate kinetics of NADH:coenzyme Q oxidoreductase isolated from bovine heart mitochondria, J. Bioenerg. Biomembr. 34 (2002) 11-19.

[39] N. Hano, Y. Nakashima, K. Shinzawa-Itoh, S. Yoshikawa, Effect of the side chain structure of coenzyme Q on the steady state kinetics of bovine heart NADH: coenzyme Q oxidoreductase, J. Bioenerg. Biomembr. 35 (2003) 257-265.

[40] D.T. Gillespie, Exact stochastic simulation of coupled chemical reactions, J. Phys. Chem. 81 (1977) 2340-2361.

[41] S. Sherwood, J. Hirst, Investigation of the mechanism of proton translocation by NADH:ubiquinone oxidoreductase (complex I) from bovine heart mitochondria: does the enzyme operate by a Q-cycle mechanism? Biochem. J. 400 (2006) 541-550.

[42] M. Ohshima, H. Miyoshi, K. Sakamoto, K. Takegami, J. Iwata, K. Kuwabara, H. Iwamura, T. Yagi, Characterisation of the ubiquinone reduction site of mitochondrial complex I using bulky synthetic ubiquinones. Biochemistry 37 (1998) 6436-644.

[43] A.D. Vinogradov, Kinetics, Control, and Mechanism of Ubiquinone Reduction by the Mammalian Respiratory Chain-Linked NADH-Ubiquinone Reductase. J. Bioenerg. Biomembr. 25 (1993) 367-375.

[44] Y. Nakashima, K. Shinzawa-Itoh, K. Watanabe, K. Naoki, N. Hano, S. Yoshikawa, The second coenzyme Q1 binding site of bovine heart NADH: coenzyme Q oxidoreductase, J. Bioenerg. Biomembr. 34 (2002) 89-94.

[45] A.D. Vinogradov, Catalytic properties of the mitochondrial NADH-ubiquinone oxidoreductase (complex I) and the pseudo-reversible active/inactive enzyme transition, Biochim. Biophys. Acta 1364 (1998) 169-185.

[46] G. Lenaz, P. Pasquali, E. Bertoli, G. Parenti-Castelli. The inhibition of NADH oxidase by the lower homologs of coenzyme Q. Arch Biochem Biophys, 169(1975) 217-26. 
[47] T. Ohnishi, J.E. Johnson Jr., T. Yano, R. Lobrutto, W.R. Widger, Thermodynamic and EPR studies of slowly relaxing ubisemiquinone species in the isolated bovine heart complex I, FEBS Lett. 579 (2005) 500-506.

[48] S. Magnitsky, L. Toulokhonova, T. Yano, V.D. Sled, C. Hägerhäll, V.G. Grivennikova, D.S. Burbaev, A.D. Vinogradov, T. Ohnishi, EPR characterization of ubisemiquinones and iron-sulfur cluster N2, central components of the energy coupling in the NADH-ubiquinone oxidoreductase (complex I) in situ, J. Bioenerg. Biomembr. 34 (2002) 193-208.

[49] R. Baradaran, J.M. Berrisford, G.S. Minhas, L.A. Sazanov, Crystal structure of the entire respiratory complex I. Nature 494 (2013) 443-48

[50] C. Hunte, V. Zickermann, U. Brandt, Functional modules and structural basis of coformational coupling in mitochondrial complex I, Science 329 (2010) 448-451.

[51] Heiske M. PhD thesis : http://www.bu.u-bordeaux2.fr/babordplus.php?special=these 


\section{Legend to the Figures.}

Figure 1: Mechanisms associated to the rate equations. MAL means Mass Action Law. In ER-HMM (Extended Reversible-Henri-Michaelis-Menten) and in CK (Convenient Kinetics) mechanisms, ENH means E-NADH, ENHQ: E-NADH-Q, ENHQH 2 : E-NADH-QH 2 , ENQ: E-NAD-Q, ENQH 2 : E-NAD-QH ${ }_{2}, \mathrm{EQH}_{2}: \mathrm{E}_{2} \mathrm{QH}_{2}$ and EN: E-NAD. In EMA mechanism 1, E* symbolizes an Enzyme-Substrates-Products complex. In mechanism 2, $\mathrm{E}_{\mathrm{A}}$ and $\mathrm{E}_{\mathrm{B}}$ represent two enzyme conformations able to bind respectively the substrates only and the products only. The derivations of EMA equation are detailed in Supplementary Materials S8.

Figure 2: Comparison of different rate equation to fit part of the experimental results. The kinetic parameters are determined by minimizing the root mean square deviation (RMSD) calculated between all experimental data and the corresponding theoretical points evaluated with the equation under study (see table 1) as explained in the Materials and Methods section. Note that the fitting procedure is performed on all experimental results represented in Supplementary Materials, but that only a small part of them is represented in this comparison for clarity. The experimental points are in black and the fitting curves in red.

(a) and (b): the experimental points are fitted by the NET equation.

(c) and (d): the experimental points are fitted by the MAL equation.

(e) and (f): the experimental points are fitted by the ER-HMM equation.

$(\mathrm{g})$ and $(\mathrm{h})$ : the experimental points are fitted by EMA equation.

The parameters for tracing the theoretical curves are listed in table 1.

Other parameters. NET: $\mathrm{k}_{\mathrm{CI}}=6.6$ nmoles $/ \mathrm{min} / \mathrm{mg}$; MAL: $\mathrm{k}_{\mathrm{f}}=3.16$ nmoles. $(\mu \mathrm{M})^{-2} \cdot \mathrm{min}^{-1} \cdot \mathrm{mg}^{-1}$; $\mathrm{k}_{\mathrm{b}}=2.0410^{-11}$ nmoles. $(\mu \mathrm{M})^{-2} \cdot \mathrm{min}^{-1} \cdot \mathrm{mg}^{-1}$.

NADH variable : (O) $\mathrm{Q}=1.1 \mu \mathrm{M} ;(\Delta) \mathrm{Q}=4.4 \mu \mathrm{M} ;(\diamond) \mathrm{Q}=10.9 \mu \mathrm{M} ;(\square) \mathrm{Q}=32.7 \mu \mathrm{M}$ Q variable: $(\mathrm{X}) \mathrm{NADH}=0.95 \mu \mathrm{M} ;(\mathrm{O}) \mathrm{NADH}=3.8 \mu \mathrm{M} ;(\Delta) \mathrm{NADH}=6.65 \mu \mathrm{M} ;(\diamond) \mathrm{NADH}$ $=14.25 \mu \mathrm{M} ;(\square) \mathrm{NADH}=28.5 \mu \mathrm{M}$.

Figure 3 Products inhibition.

Figure 2-a: NAD inhibition with $\mathrm{NADH}=38 \mu \mathrm{M}$ and $\mathrm{Q}=69 \mu \mathrm{M}$ (See also figures $\mathrm{z}$ to ac in the Supplementary Materials. This figure corresponds to figure ac with ER-HMM equation). Figure 2-b: $\mathrm{QH}_{2}$ inhibition with $\mathrm{NADH}=28.5 \mu \mathrm{M}$ and $\mathrm{Q}=54.5 \mu \mathrm{M}$

(See also figures $\mathrm{v}$ and $\mathrm{w}$ in the Supplementary Materials. This figure corresponds to figure $\mathrm{w}$ with ER-HMM equation).

The fitting curve is represented by a continuous red line.

\section{Figure 4: Complex I inhibition by the substrate Q.}

The curves are drawn according to the ER-HMM equation (eq.13) with the parameters of table 2 in the absence of Q substrate inhibition (- - ) and with the term Is $=1+\mathrm{Q} / \mathrm{K}_{\mathrm{I}}$ (red large line) with the parameters of table 3 , second row $\left(\mathrm{k}_{\mathrm{f}}=2194, \mathrm{~K}_{\mathrm{NADH}}=4.5 \mu \mathrm{M}, \mathrm{K}_{\mathrm{Q}}=14.7 \mu \mathrm{M}\right.$, $\mathrm{K}_{\mathrm{NAD}}=743 \mu \mathrm{M}, \mathrm{K}_{\mathrm{QH} 2}=7.5 \mu \mathrm{M}$ and $\left.\mathrm{K}_{\mathrm{I}}=576 \mu \mathrm{M}\right)$ or with the term Inc $=1 /\left(1+\mathrm{Q} / \mathrm{K}_{\mathrm{I}}\right)($ thin black line overlapped by the red one) with the parameters of table 3, third row $\left(\mathrm{k}_{\mathrm{f}}=2254\right.$, $\mathrm{K}_{\mathrm{NADH}}=4.5 \mu \mathrm{M}, \mathrm{K}_{\mathrm{Q}}=15.1 \mu \mathrm{M}, \mathrm{K}_{\mathrm{NAD}}=743 \mu \mathrm{M}, \mathrm{K}_{\mathrm{QH} 2}=7.6 \mu \mathrm{M}$ and $\left.\mathrm{K}_{\mathrm{I}}=561 \mu \mathrm{M}\right)$. The black diamonds correspond to the experimental points at (a) $\mathrm{NADH}=28.5 \mu \mathrm{M}$, (b) $\mathrm{NADH}=47.5 \mu \mathrm{M}$. (c) depicts the rate as a function of $\mathrm{QH}_{2}$ with $\mathrm{NADH}=28.5 \mu \mathrm{M}$ and $\mathrm{Q}=$ $45.5 \mu \mathrm{M}$ with steric inhibition ( - ) or non competitive inhibition ( - superimposed with the previous one - ) or without any inhibition ( $--=)$.

The fit of the other inhibitory function are very similar (not shown). See also figures S5 and S6 in Supplementary Materials. 


\title{
Modelling the respiratory chain complexes with biothermokinetic equations - The case of complex I
}

\author{
Margit Heiske ${ }^{1,2,4}$, Christine Nazaret ${ }^{3}$, Jean-Pierre Mazat ${ }^{1}$
}

${ }^{1}$ Université de Bordeaux, Bordeaux, France et Laboratoire de métabolisme énergétique cellulaire, IBGC - CNRS UMR 5095, 1 Rue Camille Saint Saëns, 33077 Bordeaux France.

${ }^{2}$ Institut für Biologie Theoretische Biophysik Humboldt-Universität zu Berlin, Invalidenstraße 42, Berlin,

${ }^{3}$ Institut de Mathématiques de Bordeaux, ENSTBB-Institut Polytechnique de Bordeaux

${ }^{4}$ Present address : Laboratoire d'Anthropologie Moléculaire et Imagerie de Synthèse, AMIS - CNRS UMR 5288, Université Paul Sabatier Toulouse III, 37 Allées Jules Guesde, 31073 Toulouse cedex

Keywords : Complex I, Oxidative phosphorylation, Modelling,

Highlights :

- Necessity of a simple and realistic rate equation of the respiratory complexes.

- Near Equilibrium Thermodynamics rate equation and Mass Action Law are inadequate

- Extended-Reversible Henri-Michaelis-Menten (ER-HMM) equation is appropriate.

A method is presented to insert the $\triangle \mu H^{+}$in the ER-HMM rate equation.

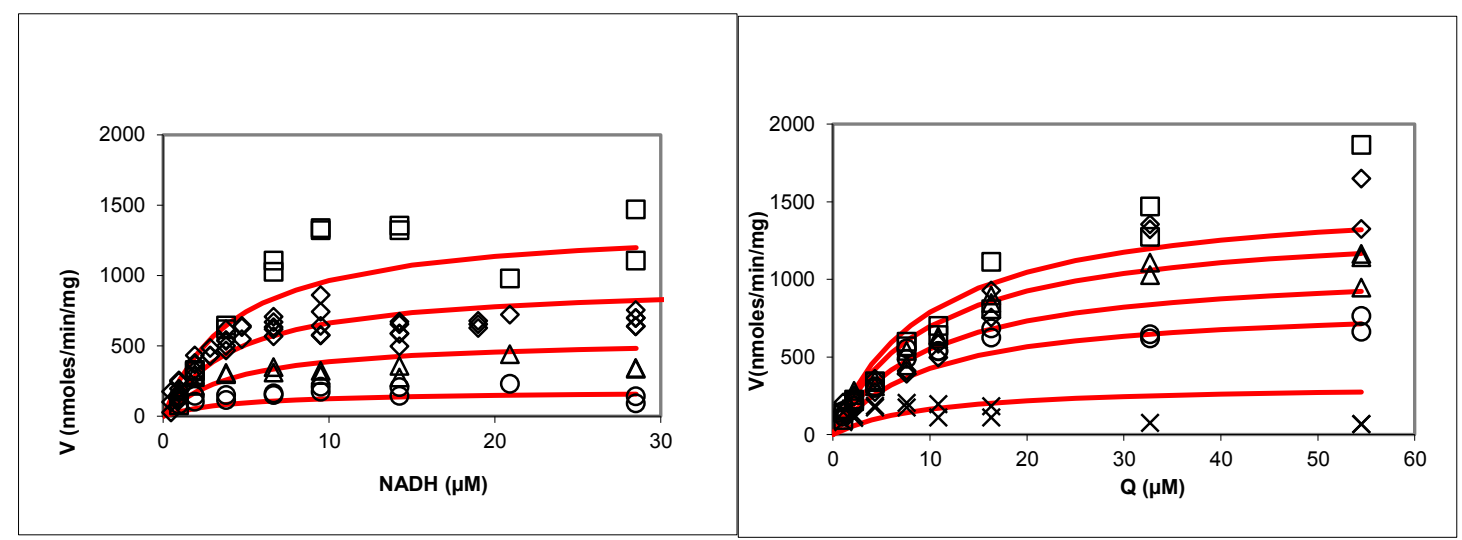




\begin{abstract}
The mitochondrial respiratory chain plays a crucial role in energy metabolism and its dysfunction is implicated in a wide range of human diseases. In order to understand the global expression of local mutations in the rate of oxygen consumption or in the production of ATP it is useful to have a mathematical model in which the changes in a given respiratory complex are properly modeled.

Our aim in this paper is to provide thermodynamics respecting and structurally simple equations to represent the kinetics of each isolated complexes which can, assembled in a dynamical system, also simulate the behaviour of the respiratory chain, as a whole, under a large set of different physiological and pathological conditions. On the example of the NADH-ubiquinol-oxidoreductase (complex I) we analyse the suitability of different types of rate equations. Based on our kinetic experiments we show that very simple rate laws, as those often used in many respiratory chain models, fail to describe the kinetic behaviour when applied to a wide concentration range. This led us to adapt rate equations containing the essential parameters of enzyme kinetic, maximal velocities and Henri-Michaelis-Menten likeconstants ( $\mathrm{K}_{\mathrm{M}}$ and $\left.\mathrm{K}_{\mathrm{I}}\right)$ to satisfactory simulate these data. Finally, we discuss a general way to introduce the electrochemical gradient into these equations leading to biothermokinetic rate equations of the respiratory complexes able to simulate the behaviour of the respiratory chain in a large variety of physiological conditions.
\end{abstract}




\section{Introduction}

The respiratory chain plays a crucial role in energy metabolism. In many cases, it consists of four enzyme complexes which are connected through two electron transporters, ubiquinone and cytochrome $c$. Three of the respiratory complexes extrude protons from the mitochondrial matrix into the intermembrane space liberating, step by step, the energy of the transfer of electrons from the low redox potential of the substrates $\mathrm{NADH}$ or $\mathrm{FADH}_{2}$ to the high redox potential of oxygen, energy then used to synthetize ATP.

It is now well documented that respiratory chain dysfunction is responsible for a wide range of human diseases including neurodegenerative diseases and cancer. Respiratory chain dysfunction may also have a possible relationship with aging [1-2] and metabolic disorders [3]. In many cases respiratory chain dysfunctions are due to mutations in the subunit constituting the respiratory chain complexes.

In order to better understand the behaviour of the respiratory chain in different physiological conditions and how the effects of pathological mutations are expressed at the global level of oxygen consumption or ATP synthesis it is useful to have a theoretical model of the respiratory chain. In other words understanding the normal or pathological interplay between these complexes and the electron transporters in the global functioning of the respiratory chain requires a model of each respiratory complex with a specific rate equation and then the integration of all the rate equations into a dynamical system representing the operation of the whole oxidative phosphorylation.

Several models, with different levels of complexity, have been established to describe the respiratory chain or its isolated complexes. It does not enter the scope of this article to review them all. We would just like to analyse the way in which the behaviour of each individual respiratory complex is approached in these models of the whole respiratory chain. It means that we discard of our analysis all the models in which the respiratory chain is represented by only one (or two depending on the electron entry point) equations (typically Magnus and Keizer [4], Cortassa et al. [5] and many others). Among the remaining models of respiratory chain two types of modelling of the individual complexes are used. The first significant models were developed in the framework of Non-Equilibrium Thermodynamic model (NET) involving a linear dependence of the flux as a function of the thermodynamic forces [6-9]. The model developed by Korzeniewski and Froncisz (1991) [10,11], is a comprehensive model also well representative of this class of thermodynamics models, extended thereafter ([12], [13]). 
Another approach, largely used to model the isolated respiratory complexes is the Mass Action Law (MAL), with the forward rate proportional to the product of substrats and the backward proportional to the product of products of the reaction. Bohnensack (1981) [14] was probably the first to derive a quantitative model of the respiratory chain involving nearly all the components of oxidative phosphorylation. In this approach the respiratory complexes were modeled according to the mass-action law. A recent example of this approach was proposed by Beard [15].

The advantage of both approaches (NET and MAL) is that they use very simple rate laws for each of the complexes with only one parameter to adjust. But these equations cannot reflect the respiratory complexes behaviours over a wide range of physiological conditions as it will be shown later in this paper.

As a matter of fact, the behaviour of the individual complexes is rather complex for several reasons: (i) the mechanisms catalysed by each separate complex are complex in themselves. The most illustrating example is the Q-cycle operating in complex III with two different quinone binding sites and the bifurcation of the electrons coming from $\mathrm{QH} 2$ molecule into two pathways. The case of cytochrome $c$ oxidase (complex IV) also involves a complex synchronisation between the 4 electrons necessary to reduce oxygen in water and the protons pumping. The case of complex I is not yet entirely resolved but already includes a cascade of 7 or 8 elementary redox reactions. (ii) not only substrates, products and modifiers play a role in the kinetics but also the electrochemical proton gradient which has an important influence on the activity of each complex and thus on the whole respiratory chain. (iii) the regulation of the activity of these complexes depends on allosteric conformations or on chemical modifications such as phosphorylation and acetylation [16-19].

On the one hand, the derivation of equations describing very precisely the underlying mechanisms of the respiratory complexes often involves a huge number of parameters which are hard or impossible to determine experimentally and will be in any case underdetermined [20-21]. On the other hand, the experimental kinetics of these complexes show, most of the time, the traditional pattern of enzymatic kinetics i.e. saturation behaviour. Furthermore, the recorded kinetics have been analysed with the usual phenomenological approaches of enzymology, particularly with the determination of $V_{\max }, K_{M}$ and $K_{I}$ at least for some of the substrates or products. It is well illustrated in the measurement of the (maximal) activity of the individual complexes, which is particularly useful in the diagnosis of mitochondrial diseases in attributing at least in some cases the pathology to a particular defect in a given complex as in [22]. In the same time the activity of the whole respiratory chain (and of part of 
TCA cycle) is recorded classically in very different conditions (resting state with a maximal transmembrane proton potential (state 4), phosphorylating state with a lower proton potential (state 3), intermediate states between states 4 and 3, uncoupled state etc.). In all these physiological conditions, the concentrations of the substrates/products of the individual respiratory complexes (NAD/NADH, Q/QH2, redox state of Cyt $c$ ) are also very different and presumably also different from the concentrations used in the determination of the (maximal) activity of the individual complexes. This could invalidate the use of the parameters values determined in these latter conditions (maximal activity determination in the absence of products) for the modelling of the whole respiratory chain functioning at non maximal activity in the presence of products.

For this reason we studied the kinetics of the isolated respiratory complexes in a broad range of substrates/products concentrations with the first aim to integrate the observations in a rate equation as simple as possible but nevertheless illustrating the main characteristics of the kinetics, particularly the saturation behaviour, the presence of products and the thermodynamic constraints. Our aim is to use these "simple" rate equations both to model kinetics of the individual isolated complexes and also the global behaviour of the whole respiratory chain when all the respiratory complexes are considered together. We will analyze different types of equations already used (NET, MAL) and we will propose other equations [23-27], more able to fit the kinetic of respiratory complexes particularly when involved in the electron transport and protons translocation through the whole respiratory chain: Extended Reversible Henri-Michaelis Menten Equation (ER-HMM), Ping Pong Mechanism (PPM), ordered mechanism (OM), "Convenience Kinetics" (CK) and a new type of equation : Extended Mass Action (EMA) (table 1 and Fig. 1). As an example, we will compare all these equations in their ability to model our kinetic results already published in [28] on beef heart complex I (NADH-Coenzyme Q reductase activity). It catalyses the reaction:

$$
\mathrm{NADH}+\mathrm{H}^{+}+\mathrm{Q} \longleftrightarrow=>\mathrm{NAD}^{+}+\mathrm{QH}_{2}
$$

Eventually we will discuss the thermodynamical aspect of the incorporation of the proton gradient in these equations, leading to thermodynamic rate equations. In this case the eatalysed reaction becomes:

$\mathrm{NADH}+5 \mathrm{H}^{+}{ }_{\text {in }}+\mathrm{Q} \longleftrightarrow \Longleftrightarrow \mathrm{NAD}^{+}+\mathrm{QH}_{2}+4 \mathrm{H}^{+}{ }_{\text {eut }}(2)$

Where $\mathrm{H}^{+}$in -means protons in the mitochondrial matrix and $\mathrm{H}^{+}{ }_{\text {out }}$-protons in the inter membrane space. 


\section{Material and Methods}

\subsection{Preparation of mitochondria}

Frozen isolated beef heart mitochondria were a gift from Dr. Joel Lunardi, prepared according to [29]. In this preparation the final mitochondrial pellets were homogenized at a concentration of $40 \mathrm{mg} / \mathrm{mL}$ and the mitochondrial suspension was frozen in liquid nitrogen as small beads of $50 \mu \mathrm{L}$ volume and stored at $-80^{\circ} \mathrm{C}$. In our experiments, one bead was thawed and diluted (1/5), aliquoted and the aliquots are frozen again. Then for each experiment an aliquot is thawed and diluted once more (1/20) in the slightly hypo-osmotic reaction buffer in order to ensure that the membranes are broken and their potential is null. For the assay itself, the mitochondrial suspension was diluted again (final concentration: $4.4 \mu \mathrm{g}$ mitochondrial proteins in $1 \mathrm{ml}$ ). All assays were performed in temperature-controlled single wavelength spectrophotometer.

\subsection{Complex I (NADH-ubiquinone oxidoreductase) assay}

The assay was performed at $37{ }^{\circ} \mathrm{C}$ according to [22] by following the decrease in absorbance at $340 \mathrm{~nm}$ resulting from the oxidation of $\mathrm{NADH}$ in $1 \mathrm{~mL}$ of medium containing $65 \mathrm{mM}$ KH2PO4 (pH 7.5), 2 mg BSA, 2 mM EDTA, $46 \mu \mathrm{M}$ antimycin A, $4.4 \mu \mathrm{g}$ mitochondrial protein and in control assays additionally $25 \mu \mathrm{M}$ rotenone. A constant ethanol concentration of $8 \mu \mathrm{L} / 1000 \mu \mathrm{L}$ was present in all assays. It means that the complement to this quantity was added when not brought by the added constituents. The concentrations of the substrates $\mathrm{NADH}$ and Q (decylubiquinone) are varied, as well as the concentrations of products $\mathrm{NAD}^{+}$ and QH2. The reaction was initiated by NADH addition. The extinction coefficient used for NADH concentration determination was $6.22 \mathrm{mM}^{-1} \mathrm{~cm}^{-1}$ at $340 \mathrm{~nm}$. The net activities have been obtained by subtracting the residual activity in the presence of rotenone from the activities without.

\subsection{Parameter estimations}

The parameters values of the different rate equations have been estimated minimizing the root mean square deviation (RMSD) between the experimental and theoretical data points. RMSD for one set of parameters was calculated simultaneously with all experimental series (see figures in the supplementary materials). To find the RMSD minimum, we used a global search routine (genetic algorithm) followed by a local one (quasi Newton based) as proposed in [30]. It permits to browse the whole parameter space and then to converge towards a well 
defined solution, usually unique in our cases. All calculations have been done using Scilab (Scilab Enterprises, 2012). Because the reaction catalyzed by the respiratory chain complex I is highly exergonic, the rate constant of the backward reaction $\mathrm{k}_{\mathrm{b}}$ is simply calculated via the Haldane relationship:

$K_{e q}=\frac{k_{f}}{k_{b}} \cdot \frac{K_{N A D}}{K_{N A D H}} \cdot \frac{K_{Q H_{2}}}{K_{Q}} \quad$ (2) or equation $K_{e q}=\frac{k_{f}}{k_{b}} \frac{C_{P}}{C_{S}}$ (3) for EMA with the equilibrium constant:

$$
K_{e q}=\exp \left(-\frac{\Delta G_{o}^{\prime}}{R T}\right)=1.54 \times 10^{+11}(4)
$$

where $\mathrm{R}=8.314 \mathrm{~J} / \mathrm{mol} / \mathrm{K}$. The temperature in the experiments was $\mathrm{T}=310 \mathrm{~K}$ and $\Delta \mathrm{G}^{\prime} \mathrm{o}=-$ $66.4 \mathrm{~kJ} / \mathrm{mol}$ at $\mathrm{pH} 7.5(69.4$ at $\mathrm{pH} 7.0)$.

\section{RESULTS}

\section{Non-saturable rate equations}

\section{I-1 Near Equilibrium Thermodynamics (NET) Equation}

In the near equilibrium approach the reaction velocity is assumed to be proportional to the Gibbs energy.

$v_{C I}=-k_{C I} \Delta G_{C I}$

with :

$$
\Delta G_{C I}=\Delta G_{o, C I}^{\prime}+R T \cdot \operatorname{Ln}\left[\frac{[N A D] \cdot\left[Q H_{2}\right]}{[N A D H] \cdot[Q]}\right] \quad \text { (6) where }[\mathrm{X}] \text { denotes the concentration of }
$$

metabolite X.

This type of equation was proposed by Westerhoff [7] and used by Korzeniewski et al. [10] for instance to model complexes I and III of respiratory chain.

The major advantage of this equation is its simplicity, since there is only one parameter to determine, besides the standard Gibbs energy which is known for the respiratory chain complexes. Furthermore one can be certain that the flux vanishes at equilibrium (for $\Delta G_{t T}=$ $\theta)$. Another point is that the equation can deal with any stoichiometric factor, also non-integer ones, which can be advantageous e.g. for the more complex stoichiometries of complex III and IV of the respiratory chain. Finally, in this equation, it is easy to take into account the proton motive force $\Delta \mu_{H}{ }^{+}$as an extra term added to $\Delta G_{\mathrm{CI}}$ of the chemical reaction [10]. 
The fact that the concentrations appear as a logarithmical term limits the increase of velocity with increasing substrate concentration. This does not correspond to a typical saturation behaviour of an enzyme but the non- linear increase of velocity somehow approaches this property, as we can see in Fig. 2 a and $b$. However, the logarithmic expression of the cencentrations leads to a very high impact of very small substrate concentrations (near zero) on the velocity, i.e. at low substrate concentration, a small change results in a great velocity ehange, and on the other side at higher concentrations, the velocity hardly changes, giving nearly overlapping curves when the other substrate is varied. As shown on Fig. $2 a$ and $b$, the initial velocities measured experimentally in the absence of products cannot be fitted correctly with this equation. It is predictable because in absence of products and at $\Delta \mu_{H}{ }^{+}=0$ with a $\Delta G^{\circ}{ }_{H}=-66.4 \mathrm{~kJ} / \mathrm{mol}$ this reaction is always very far from equilibrium.

\section{I-2 The Mass Action Law equation.}

The MAL equation applied to the enzymatic reaction (1) reads:

$v_{C I}=k_{f}[N A D H] .[Q]-k_{b}[N A D] .\left[Q H_{2}\right]$

or introducing $\mathrm{K}_{\mathrm{eq}}: v_{C I}=k_{b}\left(K_{e q}[N A D H] .[Q]-[N A D] .\left[Q H_{2}\right]\right)$

This type of equation was largely used for modelling the respiratory chain complexes (e.g. [14-15]). As previously, introducing the $K_{e q}=k_{f} / k_{b}$ allows one to be sure that $v_{C I}$ vanishes at equilibrium. Additional terms in the form of $\exp \left[\mathrm{n}_{l} \mathrm{RT} \ln \left(\left[\mathrm{H}^{+}{ }_{\text {in }}\right] /\left[\mathrm{H}^{+}{ }_{\text {out }}\right]\right)\right]$ can be added (as in [15]) to take the proton motive force into account where $n_{I}$ is the number of protons expelled by complex $\mathrm{I}$. The equation has the same amount of parameters as the NET equation and is also not limited to particular stoichiometric factors.

A rather cumbersome drawback of this equation is that the velocity increases proportionally to the substrate concentrations. This does not correspond to the typical saturation behaviour of enzymes (see Fig. $2 \mathrm{c}$ and $d$ ). This is the main reasen for which this equation fails to fit the initial velocities measured for different substrate concentrations.

We conclude that these equations (see supplementary materials S1), MAL as well as the NET equation cannot be used for an accurate description of respiratory complexes kinetics when there is a possible variation in substrates and products concentrations. It is necessary to introduce a saturation term in the rate equations.

\section{The saturable rate equations.}




\section{II-1 The equations}

The list of the rate equations tested in this work which exhibit a saturable pattern as a function of substrate or product concentrations are listed in table 1 (rows 3 to 7 ) and their mechanism depicted in Fig. 1. They all involve the same type of numerator as MAL equation vanishing at equilibrium (or an equivalent expression in the case of EMA). In addition, they also involve a denominator with Michaelis constants $\mathrm{K}_{\mathrm{M}}$ for substrates and products. Because we look for an equation as simple as possible we assume that the $\mathrm{K}_{\mathrm{M}}$ for a substrate/product is independent of the binding of the others which is not necessarily true in the reality. Because the denominators of the equations are different, one can predict that the values of $K_{M}$ may be different for the different equations. All these equations but PPM are equivalent in absence of products.

\section{Table 1 and Fig. 1}

The Extended-Reversible Henri-Michaelis-Menten (ER-HMM) equation [23-25; 31-34] corresponds to a random binding/release of substrates/products as represented in Fig. 1. It is well described in all textbooks (see [26] for instance).

The convenience kinetics equation (CK) is a generic equation which can account for any number of substrates and products. It has been proposed by Liebermeister and Klipp [27] and includes the same basic kinetic parameters (linked by the same Haldane relationship) than ER-HMM equation above but arranged slightly differently in the denominator so that the Km values may be different. In the case of 2 substrates 2 products, it also corresponds to a random binding mechanism but, in contrast to ER-HMM mechanism, without the non-reactive ternary complexes E-NADH-QH2 and E-NAD-Q (see Fig. 1).

The interests of ER-HMM equation is well explained by Cornish-Bowden and Hofmyer in [23] which also applies to CK: minimal number of parameters, adherence to thermodynamic constraints, competition between substrates and their corresponding products and saturability.

The equation given in table 1 for the order mechanism (OM) correspond to the case NQNQ meaning that NADH binds first then $\mathrm{Q}$ and NAD is released first then $\mathrm{QH}_{2}$. The four possible sequences of substrates binding/products release have been tested (see table 2).

A ping-pong mechanism was also proposed for complex I kinetics (see the discussion in [28]). It can correspond to two situations: NNQQ or QQNN meaning that either NADH reacts first 
and gives $\mathrm{NAD}$ and then $\mathrm{Q}$ gives $\mathrm{QH}_{2}$ or vice versa. In the simplified form where the $\mathrm{K}_{\mathrm{M}}$ are independent of each other, both equations have the same expression given in table 1.

Finally we tested EMA equation (Extended Mass Action) which is based on mass action according to two different possible mechanisms (see supplementary materials S7 for a complete derivation of this equation). It can also be seen as a simplified ER-HMM equation in which the Michaelis constants of the substrates (resp. the products) are fused in one constant $\mathrm{C}_{\mathrm{S}}\left(\right.$ resp. $\mathrm{C}_{\mathrm{P}}$ ). The equation has only 4 parameters which are not independent; one can be replaced by the equilibrium constant via the Haldane-like relationship:

$K_{e q}=\frac{k_{f}}{k_{b}} \frac{C_{P}}{C_{S}}(3)$

This equation has the big advantage that any stoichiometric factor (also non integer) can be included, like for the NET and MAL equations. This equation was developed independently by Liebermeister et al. [35].

For each equation, the results of parameters fitting using all the experimental points are listed in table 2. Fig. 2e to h gives an example of these fittings in the case of ER-HMM and EMA equation with only some of the experimental points. The figures showing the results of the fittings according to other saturable rate laws with the complete set of experimental points are shown in supplementary materials. The results are always plotted in comparison with the ERHMM fit (blue dashed).

\section{Table 2 and Fig. 2}

Comparing the RMSD (table 2), it is clear that all equations but EMA give nearly the same good accuracy. The rate constants and the substrates $\mathrm{K}_{\mathrm{M}}$ values are also very similar (except for EMA for which the parameters have different meanings). An average value of $k_{f}=1810 \pm$ 43 nmoles. $\min ^{-1} \cdot$ prot $^{-1}, \mathrm{~K}_{\mathrm{NADH}}=4.6 \pm 0.7 \mu \mathrm{M}$ and $\mathrm{K}_{\mathrm{Q}}=10.2 \pm 1.3 \mu \mathrm{M}$ are obtained. This is not the case for the $K_{M}$ values of the products. The determination of the products $K_{M}$ is more difficult because as mentioned above, the complex I reaction is largely irreversible in the absence of $\Delta \mu_{\mathrm{H}}{ }^{+}$. The only way to have an indication of the products $\mathrm{K}_{\mathrm{M}}$ is to record the reaction rate in the forward direction in the presence of different concentrations of the products (Fig. 3). The values of the products $\mathrm{Km}$ are dependent on the structure of the denominator of each equation, but still one can state that $\mathrm{K}_{\mathrm{NAD}}$ is clearly high and difficult to measure indicating a low affinity of $\mathrm{NAD}^{+}$. On the other hand $\mathrm{K}_{\mathrm{QH} 2}$ is rather low, sometimes 
lower than $\mathrm{K}_{\mathrm{Q}}$. Both $\mathrm{K}_{\mathrm{m}}$ products depend upon the rate equation used to fit the experimental results.

\section{Figure 3 a et $b$}

\section{II-2 Q inhibition}

Figure 4 shows a clear inhibition at high Q concentration (> $100 \mu \mathrm{M})$. Different type of substrate inhibitions can be considered such as non-competitive, substrate/steric inhibition (at the normal binding site). On the example of ER-HMM equation, several inhibition terms have been tested. The ER-HMM equation reads then:

$v=E_{t} I_{n c} \frac{k_{f} \cdot \frac{N A D H}{K_{N A D H}} \cdot \frac{Q}{K_{Q}}-k_{b} \cdot \frac{N A D}{K_{N A D}} \cdot \frac{Q H_{2}}{K_{Q H_{2}}}}{\left(1+\frac{N A D H}{K_{N A D H}}+\frac{N A D}{K_{N A D}}\right)\left(1+\frac{Q}{K_{Q}} I_{S}+\frac{Q H_{2}}{K_{Q H_{2}}} I_{S}\right)}$

with the inhibition terms $I_{s}$ and $I_{n c}$ described in the following.

\section{Substrate-product or steric inhibition $I_{S}$}

This type of inhibition corresponds to the obstruction of the catalytic site of quinone reduction by quinone or quinol. Assuming that $\mathrm{Q}$ and $\mathrm{QH}_{2}$ obstruction has the same effect on the binding of both molecules $\mathrm{QH}_{2}$ or $\mathrm{Q}$, the term $\mathrm{I}_{\mathrm{s}}$ can be written:

$I_{S}=1+\frac{[Q]}{K_{i 1}}+\frac{\left[Q H_{2}\right]}{K_{i 2}}$

where $\mathrm{K}_{\mathrm{i} 1}$ and $\mathrm{K}_{\mathrm{i} 2}$ are the inhibition constants of $\mathrm{Q}$ and $\mathrm{QH}_{2}$ respectively. In the case where $\mathrm{K}_{\mathrm{i} 1}=\mathrm{K}_{\mathrm{i} 2}=\mathrm{K}_{\mathrm{i}}$, the expression (10) becomes:

$I_{S}=1+\frac{\left[Q_{t o t}\right]}{K_{i}}$ where $\left[\mathrm{Q}_{\mathrm{tot}}\right]=[\mathrm{Q}]+\left[\mathrm{QH}_{2}\right](11)$

\section{Non competitive inhibition $I_{n c}$}

Another possibility is that $\mathrm{Q}$ or/and $\mathrm{QH}_{2}$ bind to a second quinone site on the molecule, modifying the activity of complex I, giving rise to a non-competitive inhibition.

The generic expression of a non-competitive inhibition is: 
$I_{n c}=\left(\frac{1}{1+\frac{[Q]}{K_{i 1}}+\frac{\left[Q H_{2}\right]}{K_{i 2}}}\right)^{n}$

The values $n=1$ and $n=2$ have been tested with inhibition by $Q_{\text {tot }}$ (with $K_{i 1}=K_{i 2}=K_{i}$ ) or $Q$ alone.

All the experimental points have been fitted by ER-HMM equation (9) involving different $I_{s}$ or $\mathrm{I}_{\mathrm{nc}}$ terms listed in table 3 (see supplementary materials Fig. S6 and S7).

Very similar accuracies of the different fits are obtained. In the case of steric inhibition, taking $\mathrm{Q}$ and $\mathrm{QH}_{2}$ separately or $\mathrm{Q}_{\text {tot }}$ as inhibitor makes no real difference (not shown). It is the same in the case of non-competitive inhibition. In this latter case taking $n=1$ or $n=2$ gives similar fits. The steric (red broad line) and non-competitive (black thin line) inhibitions are represented on figure 4 and overlap. The curve without inhibition (blue dashed line) is also shown for comparison. Interestingly the values of $\mathrm{K}_{\mathrm{Q}}$ (and of $\mathrm{K}_{\mathrm{NADH}}$ ) remain approximately the same for all types of inhibition. Only $\mathrm{K}_{\mathrm{QH} 2}$ changes significantly when part of the normal (product) $\mathrm{QH}_{2}$ inhibition is taken by the specific inhibitory mechanism.

\section{Table 3 and Fig. 4}

\section{H - Incorporation of the $\triangle \mu H^{+}$. Towards a biothermodynamies rate equation for the respiratory complexes.}

Until now we considered the chemical reaction:

$\mathrm{NADH}+\mathrm{H}^{+}{ }_{\text {in }}+\mathrm{Q} \Leftrightarrow \mathrm{NAD}^{+}+\mathrm{QH}_{2}-(1)$

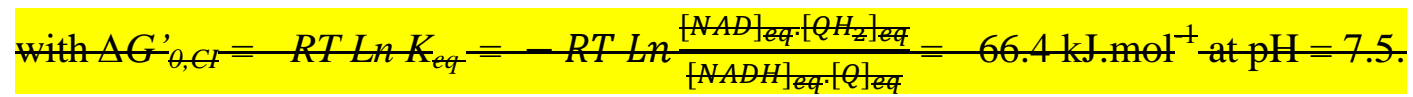

Thus the $\Delta G^{\prime}{ }_{\mathrm{CI}}$ of the chemical reaction at $\mathrm{pH} 7.5$ is:

$\Delta G^{\prime}{ }_{C I}=\Delta G^{\prime}{ }_{Q, C I}+R T \operatorname{Ln} \frac{[\mathrm{NAD}]_{-} \cdot\left[Q \mathrm{QH}_{2}\right]_{=}}{[\mathrm{NADH}]_{-} \cdot[Q]_{-}}$(7)

These equations above are valid in absence of proton gradient, i.e. in conditions for which the protons equilibrate rapidly on either side of the membrane supporting the respiratory complex. In intact mitochondria, protons are pumped by the respiratory complexes and create a proton gradient which opposes the protons extrusions and thus slows down the chemical reaction. In these conditions, as noticed in the introduction, the complex I reaction reads:

$\mathrm{NADH}+\mathrm{H}^{+}{ }_{\text {in }}+\mathrm{Q}+4 \mathrm{H}^{+}{ }_{\text {in }} \Leftrightarrow \mathrm{NAD}^{+}+\mathrm{QH}_{2}+4 \mathrm{H}^{+}{ }_{\text {out }}$ (2) 
(the first proton in the left part of the reaction is the sealar protons accompanying the chemical reaction). The four other protons are the vectorial protons transduced from the matrix towards the intermembrane space.

From the thermodynamical point of view this corresponds to an opposite force:

$\Delta G H=R T \operatorname{Ln}\left(\left[H^{+}{ }_{\text {in }}\right] / 10^{-7.5}\right)-4 \Delta \mu_{H_{+}-}$with $\Delta \mu_{H_{+}}=F \Delta \psi_{\text {in-out }}+\mathrm{RT} \operatorname{Ln}\left(\left[\mathrm{H}^{+}{ }_{\text {in }}\right] /\left[\mathrm{H}^{+}{ }_{\text {out }}\right]\right)(14)$

$F$ is the Faraday's constant, $\Delta \psi_{\text {in out }}$ is the membrane potential measured as the inner potential minus the outer potential. $\Delta \mu_{H_{+}}$is thus usually negative so that the term $\Delta G H$ is $>0$ for physiological values $\left(\mathrm{pH}_{\mathrm{in}}>7.5\right)$. With these notations, the free energy of reaction $(2) \Delta G^{\mathrm{H}} \in$; taking into account the movement of protons and the internal and external $\mathrm{pH}$ is: $\Delta G^{\mathrm{H}}{ }_{\mathrm{Cl}}=\Delta \mathrm{G}_{\mathrm{Cl}}^{\prime}+\Delta \mathrm{GH}=\Delta \mathrm{G}_{\mathrm{Cl}}^{\prime}-\mathrm{RT} \ln \left(\left[\mathrm{H}_{\mathrm{in}}^{+}\right] / 10^{-7.5}\right)-4 . \Delta \mu_{\mathrm{H}}^{+}$

This corresponds to a new equilibrium constant when $\Delta \mu_{H}^{+} \neq 0$.

$\left.\mathrm{K}_{\mathrm{eq}}^{\mathrm{H}}=\operatorname{Exp}\left(\left(\Delta \mathrm{G}_{0, \mathrm{CI}}{ }^{+}+\Delta \mathrm{GH}\right) / \mathrm{RT}\right)=\operatorname{Exp}\left(\Delta \mathrm{G}_{0, \mathrm{CI}}{ }_{\mathrm{C}} / \mathrm{RT}\right) \cdot \operatorname{Exp}(\Delta \mathrm{GH}) / \mathrm{RT}\right)$

or $\left.K_{\text {eq }}^{H}=K_{\text {eq }} \cdot \operatorname{Exp}(\Delta G H) / R T\right)=K_{\text {eq }} \cdot e^{-\alpha}$ with $\alpha=\Delta G H / R T \quad(\alpha>0)$

Thus all is as if the equilibrium constant $K_{\text {eq }}$ - of the chemical reaction was changed in $K_{\text {eq }}^{\mathrm{H}}$ by introducing the electrochemical proton gradient. This corresponds well to the displacement of the chemical reaction under the action of the proton gradient.

According to Haldane's relationship,

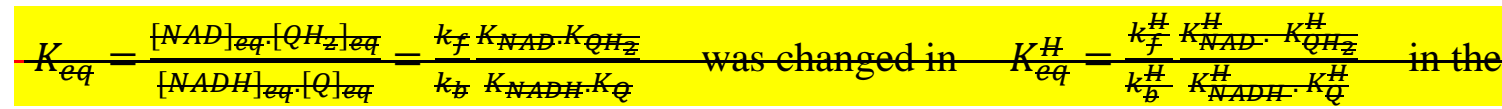
presence of $\mathrm{H}^{+}$gradient.

$\mathrm{K}_{\mathrm{eq}}$ is a function of $\mathrm{k}_{\mathrm{f}}, \mathrm{k}_{\mathrm{b}}, \mathrm{K}_{\mathrm{NAD}}, \mathrm{K}_{\mathrm{Q}}, \mathrm{K}_{\mathrm{NADH}}, \mathrm{K}_{\mathrm{QH} 2}$. The proton gradient can in principle affect all of these values leading to new values $\mathrm{k}_{\mathrm{f}}^{\mathrm{H}}, \mathrm{k}_{\mathrm{b}}^{\mathrm{H}}, \mathrm{K}^{\mathrm{H}}{ }_{\mathrm{NAD}}, \mathrm{etc}$. One can hypothese as a first approximation, that it only affect the rate constants $k_{f}$ and $k_{b}$ according to the expression:

$\mathrm{k}_{\mathrm{f}}^{\mathrm{H}}=\mathrm{k}_{\mathrm{f}} \cdot \mathrm{e}^{-\zeta \alpha}$ and $\mathrm{k}_{\mathrm{b}}^{\mathrm{H}}=\mathrm{k}_{\mathrm{b}} \cdot \mathrm{e}^{(1-\zeta) \alpha}-(15)$,

where $1 \geq \zeta \geq 0$, is a parameter which splits the proton gradient between both rate constants. The $\zeta$ value will depend upon the rate of OxPhos at steady state measured in condition of known $\Delta \mu_{H}{ }^{+} \neq 0$. It may be different for each complex, 


\section{DISCUSSION AND CONCLUSION}

\section{NET and MAL rate equations}

As obvious in Fig. 2 (a to d), Near Equilibrium Thermodynamics (NET) and Mass Action Law (MAL) rate equations fail to adequately represent the activity of complex I when the concentrations of substrates are varied. In the case of NET, the logarithmic term dampens the variations of substrates concentrations so that the rate value is nearly constant (between 540 and $650 \mathrm{nmol} / \mathrm{min} / \mathrm{mg}$ prot) over the nearly two order of magnitude of substrates concentrations used. Only at very low concentration of one substrate, the rate decreases rapidly toward zero (In fact to minus infinity; see the red line along the y-axis in Fig.2 a and b). It is not surprising because in conditions of very low products concentrations $(0.1 \mu \mathrm{M}$ each in our case to make the calculation possible) with a value of $\Delta \mathrm{G}^{\prime}{ }_{\mathrm{o}}=-66.4 \mathrm{~kJ}$, the reaction is far from equilibrium and its rate will not obeys the conditions of near equilibrium thermodynamics.

A slightly better fit is obtained with MAL equation, because in this case the rate equation is sensitive to substrates concentrations variations. However the linear increase of the rate as a function of substrates concentrations does not correspond to the reality of saturable kinetics. For comparison the fit of the same points with a typical saturable kinetic equation (ER-HMM, Fig. 2 e-f) is shown. However NET and MAL equations may be a good choice for the description of the respiratory chain in conditions close to equilibrium which can be the case for complex I, II and III of respiratory chain in in vivo conditions with $\Delta \mu_{\mathrm{H}^{+}} \neq 0$. It must be stressed that in all cases, the constants involved in these equations $\left(\mathrm{k}_{\mathrm{CI}}\right.$ or $\left.\mathrm{k}_{\mathrm{f}}\right)$ get ad hoc values which do not correspond to any intrinsic property (kinetic constants) of the respiratory complexes.Nevertheless, as demonstrated by Pillay et al. [36], a MAL model can give rise to a "saturation" behaviour particularly in redox cycles when the sum of the redox couples (NAD/NADH and Q/QH2) is constant. In these conditions, the maximal velocity depends upon the rate constant and the total concentrations of substrates/products and the half saturation concentration also depends upon the total concentration of the redox couples and is thus variable and different of a real $\mathrm{Km}$. Using such equations will make it difficult to analyze the effect on OXPHOS of a variation of a particular kinetic parameter ( $\mathrm{k}_{\mathrm{cat}}$ or $\mathrm{Km}$ ) of a given respiratory complex or of physiological or pathological changes in total substrate and product concentrations. 
On the contrary Fig. 2 e-h (and Fig. S2 to S7 in supplementary materials) and table 2 show that all the other equations tested in our study give similar good fits when compared with the whole set of our experimental data. It should be however noticed that EMA fit, which involves two parameters less, is less accurate mainly because it does not have independent saturation terms for each of the substrates/products. Their binding is rather considered simultaneous (see Supplementary Materials) and quantified by the phenomenological constants Cs and $\mathrm{Cp}$. When the Km of substrates (respectively the products) are similar the phenomenological constants $\mathrm{C}$ represent adequately their association by an average constant. It is no longer the case when the Km are different as here for NAD and NADH.

\section{Kinetic mechanism and kinetic constants}

The striking results of this study that several kinetic equations are equally able to fit the experimental results over large concentrations of substrates and products is consistent with the fact that the kinetic mechanism of this reaction is still a matter of discussion. It means that this form of analysis does not shed light on the, too complex, kinetic mechanism of the reaction. Fato et al. [37] proposed a ping-pong mechanism in the case of mitochondria isolated from bovine heart with the oxidation of NADH preceding the reduction of ubiquinone. Nakashima et al. [38] used $\mathrm{CoQ}_{1}$ as the electron acceptor to analyze the activity of complex I purified from bovine heart. They proposed an ordered sequential mechanism with $\mathrm{CoQ}_{1}$ binding as the first step and $\mathrm{CoQ}_{1} \mathrm{H}_{2}$ releasing as the last step. Hano et al. [39] assumed that the kinetics of complex I obeys an ordered sequential mechanism when they used decylubiquinone (DQ) as the electron acceptor.

Analyzing the same set of experimental results as in this study in the light of a stochastic model based on Gillespie approach [40] and taking into account the structure (distances) and the midpoint potentials of the reaction centers we showed that the kinetics may not necessarily obey a simple mechanism (ordered or ping-pong) [28]. This is particularly due to the substantial distance (around $90 \AA$ ) between NADH oxidation site and quinone reduction site and the presence of seven redox reactions in between. It makes the two extreme redox sites (NADH/NAD on the one hand and Q/QH2 on the other hand) as if they were independent from each other. The stochastic simulations also evidenced a plateau for saturating NADH concentrations (see supplementary figures a-j). It renders the fit by any equation used in this study slightly inaccurate: at high NADH concentrations the theoretical curves go on increasing weakly, while the experimental rates (as well as the stochastic simulations) are steady and quasi horizontal. We showed that this does not result from a 
substrate inhibition but simply from the accumulation of electrons in the intermediate redox centers as NADH concentration increases, leading to a sort of electron buffering effect [28]. This can be easily understood in the following way: if the rate of the second half of the mechanism $(\mathrm{Q}=>\mathrm{QH} 2)$ is low as compared to first one (NADH $\Rightarrow$ NAD) and to the intermediate redox reactions inside complex I, for instance low Q and high NADH, then the electrons accumulate progressively backwards on the 7-8 FeS centers in between the two half reactions. As soon as one $\mathrm{QH} 2$ molecule is formed, two electrons from some FeS centers are again rapidly available. The limiting step is the release of the previously formed QH2 molecule and the binding of the new Q molecule which are phenomena much slower than the "flitting" of the electrons between the FeS centers and operating at constant rate when Q and $\mathrm{QH}_{2}$ are constants. In other words, when the second half reaction is slower than the first one, there are always two electrons to reduce a $\mathrm{Q}$ molecule when present. It is the reason why a plateau is observed in the kinetics particularly at high NADH and low Q.

Another salient feature of our study is that for all the saturable equations (but EMA) the maximal rate constants and the $\mathrm{Km}$ are nearly the same (see table 2) and well comparable with the data in the literature on beef heart mitochondria complex I. For instance we find a forward maximal rate constant between 1773 and $1910 \mathrm{nmol} \mathrm{NADH}$. $\mathrm{min}^{-1} \cdot \mathrm{mg}^{-1}$. Fato et al. [37] determined on submitochondrial particles for different types of quinone a $\mathrm{k}_{\mathrm{cat}}$ ranging from 170 to $1560 \mathrm{nmol} \mathrm{NADH}$. $\mathrm{min}^{-1} \cdot \mathrm{mg}^{-1}$. Sherwood and Hirst [41] reported a value of 3 $100 \mathrm{nmol}$ NADH. $\mathrm{min}^{-1} \cdot \mathrm{mg}^{-1}$ and Hano et al. [39 ] a value of $1860 \mathrm{nmol} \mathrm{NADH} \cdot \mathrm{min}^{-1} \cdot \mathrm{mg}^{-1}$. Lower values around $500 \mathrm{nmol} \mathrm{NADH} . \mathrm{min}^{-1} \cdot \mathrm{mg}^{-1}$ were reported by other authors with different quinone analogs [42].

$\mathrm{K}_{\mathrm{m}}(\mathrm{NADH})$ was found in the range of $0.6-2.7 \mu \mathrm{M}$ on isolated complex $\mathrm{I}$ in the presence of different quinone types, among them also decylubiquinone [38, 39], always in beef heart mitochondria. For SMP a value of $9.2 \mu \mathrm{M}$ has been found [37]. Vinogradov [43] reported for $\mathrm{K}_{\mathrm{m}}(\mathrm{NADH}) 7.6 \mu \mathrm{M}$ and $7.2 \mu \mathrm{M}$ for coupled and uncoupled SMPs, respectively. Nakashima et al. [38] found a $\mathrm{K}_{\mathrm{m}}(\mathrm{NADH})$ around $2 \mu \mathrm{M}$ depending of Q1 and NAD concentrations. It is well comparable with the values $4.2-6.1 \mu \mathrm{M}$ we found in our fits.

The values found for $\mathrm{K}_{\mathrm{m}}(\mathrm{Q})$ are more variable, due to the different type of quinone that has been used. Fato et al. [37] determined the $\mathrm{K}_{\mathrm{m}}(\mathrm{Q})$ for 7 different types of quinones on bovine submitochondrial particles. Their values for decylubiquinone (DQ) were found to be 1.8 and $2.1 \mu \mathrm{M}$. A higher value of $24 \mu \mathrm{M}$ has also been reported in [41]. For complex I isolated from beef heart, values of $4.4-12.9 \mu \mathrm{M}$ for Q1 and Q2 $[38,39,44]$ have been reported and $51 \mu \mathrm{M}$ 
[39] for decylubiquinone. These values are similar to the values between 9.5 and 13.8 found in our fits.

Less data are available with respect to the $\mathrm{Km}$ values of the products and they are more difficult to compare to our values because of their dependence on the rate equations structures. Vinogradov [45] found a $\mathrm{K}_{\mathrm{i}}$ value for NAD of $1250 \mu \mathrm{M}$ on uncoupled SMP, which lies among the K-values we found for the different equations. However, for the reverse reaction under coupled conditions the author reported a $\mathrm{Km}$ of $7.2 \mu \mathrm{M}$ for NAD. To explain the difference of about three orders of magnitude it was suggested that NAD binds to a different site for the reverse sense. But this large difference may also be due to the equation that has been used for the determination of these values, as we can see on tables 2 and 3. With only three independent parameters (instead of five for other equations ER-HMM, PPM or OM), the EMA equation is a significantly less precise description of the given data. However, for most data curves EMA is still comparable to the other rate equations, as one can see in Fig. 2 and on Fig. S2 . But since the product inhibition by $\mathrm{QH}_{2}$ and $\mathrm{NAD}^{+}$are approximately three orders of magnitude apart, it is obvious that EMA cannot describe accurately the influence of both. Here the fits lead to a good description of the influence of $\mathrm{QH}_{2}$, but in contrast, in the series where $\mathrm{NAD}^{+}$was varied the data description is not satisfactory. For the latter no $\mathrm{QH}_{2}$ was present which means that the product term in the EMA equation was always 0 , with $\mathrm{NAD}^{+}$present or not. Hence EMA is not applicable to such extreme situation

\section{Inhibition by $Q$.}

In our experimental data one can observe an inhibition of complex I at high concentrations of decylubiquinone (Fig. 4). Indeed oxidized quinones have been suspected to exert a negative effect on complex I activity at higher concentrations. Lenaz et al. [46] showed an inhibitory effect by the short chain CoQ analogue $\mathrm{Q}_{3}$ and assumed that there is need for long chain ubiquinone for a proper functioning of complex I. Other authors reported this inhibition with different types of quinones [37] and [44]. Grivennikova et al. [18] reported even a very strong inhibition by the short chain ubiquinone. This could suggest the existence of a second inhibitory site for quinone (oxidized) in addition for the substrate site as we already discussed in [28] (see also [41], [44], [47-50]. Althoug,there is no evidence of a precise second ubiquinone site, there is the possibility of ubiquinone taking several positions either in the large reaction pocket or on the way leading from the membrane to the reaction site and thus impeding or hindering the access of the ubiquinone substrate by ubiquinol or the release of the 
ubiquinol product by ubiquinone, well modeled by a steric inhibition either by Q or QH2. It should be noticed that the inhibitory concentration of $\mathrm{Q}$ are rather high $(>100 \mu \mathrm{M})$ and that $15-20 \%$ inhibition is obtained at $350 \mu \mathrm{M}$ Q. Thus the inhibition term can be presumably neglected in most of the cases.

\section{Choice of a rate equation}

Because all equations but EMA give a similar good fit of our kinetic experiments performed with large variations of the substrates and products, we select the ER-HMM equation to represent complex I activity. It is among the simplest and corresponds to the random bi-bi mechanism. As noted above, the inhibitory term is probably most of the time superfluous. If necessary, it will be added under the form of a non competitive inhibition by $\mathrm{Q}_{\text {tot }}$ (see Fig. S5 and S6). Both type of inhibition (non competitive and steric) give very comparable inhibitory pattern (Fig. S5 and S6).

$$
v=E_{t} I_{n c} \frac{k_{f} \cdot \frac{N A D H}{K_{N A D H}} \cdot \frac{Q}{K_{Q}}-k_{b} \cdot \frac{N A D}{K_{N A D}} \cdot \frac{Q H_{2}}{K_{Q H_{2}}}}{\left(1+\frac{N A D H}{K_{N A D H}}+\frac{N A D}{K_{N A D}}\right)\left(1+\frac{Q}{K_{Q}}+\frac{Q H_{2}}{K_{Q H_{2}}}\right)} \quad \text { (13) with } I_{n c}=\left(\frac{1}{1+\frac{\left[Q_{t o t}\right]}{K_{i}}}\right)
$$

We have shown that this type of equation (ER-HMM) can be used to fit the kinetics of the other complexes of the respiratory chain [51].

The introduction of the $\Delta \mu_{\mathrm{H}+}$ in these equations is then a delicate problem due to its possible stiff effects on the rate constants. $A$ thermodynamics approach just subtracting the $\Delta \mu_{\mathrm{H}+}$ from the $\Delta G$ of the chemical reaction is a way to handle this problem which was adopted by many authors $[10,15]$ and which is particularly appropriate for complex $I$. As a matter of fact, according to the $X$ ray structure of complex $\mathrm{I}$ [49-50], the electron path is entirely in the matrix arm; thus it is not in principle subject to the electrical field in the membrane. The only way to couple the transmembrane proton pumping to the energy liberated in the redox reactions is through a still unknown conformational change of the complex. It means that the $\Delta G$ of the chemical reaction will be used to build the $\Delta \mu \mathrm{H}^{+}$thus decreasing (algebraically increasing) the $\Delta G^{H}$ of the global reaction as explained in section III. This is the reason why, in a first approach, we choose to put all the $\Delta \mu \mathrm{H}^{+}$on the rate constants. This choice is also in accord with our wish to derive a rate equation as simple as possible which respects the thermodynamic constraints. Distributing the $\Delta \mu \mathrm{H}^{+}$- on all the kinetic parameters would very much complicate the rate equation and would be somewhat arbitrary. It does not mean that the 
Kms are not affected by the conformational change, but because we do not know how, we take for the moment the simplest assumption. The problem could be different for the other respiratory complexes, the complex III for instance in which the path of electron is perpendicular to the membrane and thus subject to the transmembrane electric field. In a forthcoming paper we will discuss the introduction of the proton gradient $A G^{\mathrm{H}}$-in biothermodynamic rate equations and analyze different approaches with respect to their ability to reproduce OXPHOS data under coupled conditions.

\section{ACKNOWLEDGEMENTS}

The authors are indebted to Drs. Joel Lunardi and Gaëlle Hardy for the preparation of mitochondria, to Dr. S. Ransac for fruitful discussions and to Prof. Edda Klipp for her constant support.

\section{REFERENCES}

[1] M., Frenzel, H. Rommelspacher, M. D. Sugawa, N. A. Dencher, Ageing alters the supramolecular architecture of OxPhos complexes in rat brain cortex. Exp. Gerontol. 45 (2010) 563-572.

[2] D. C. Wallace, Mitochondrial DNA mutations in disease and aging. Environ. Mol. Mutagen. 51 (2010) 440-450.

[3] S. DiMauro, E. A. Schon, Mitochondrial disorders in the nervous system. Annu. Rev. Neurosci. 31, (2008) 91-123.

[4] G. Magnus, J. Keizer.. Minimal model of b-cell mitochondrial Ca21 handling. Am. J. Physiol. 273. Cell Physiol. 42 (1997) C717-C733.

[5] S.Cortassa, M.A.Aon, E.Marban, R.L.Winslow, B. O'Rourke,. An integrated model of cardiac mitochondrial energy metabolism and calcium dynamics. Biophys J. 84 (2003) 273455 .

[6] H. Rottenberg, Non-equilibrium thermodynamics of energy conversion in bioenergetics, Biochim. Biophys. Acta 549(1979) 225-253.

[7] , H.V. Westerhoff, K. van Dam Thermodynamics and control of free-energy transduction, (1987) Elsevier, Amsterdam.

[8] J.W. Stucki,The thermodynamic-buffer enzymes. E. J. Biochem. 109 (1980) 257-267.

[9] J.W. Stucki, The optimal efficiency and the economic degrees of coupling of oxidative phosphorylation. E. J. Biochem. 109 (1980) 269-283. 
[10] B. Korzeniewski, W. Froncisz, Theoretical studies on the control of the oxidative phosphorylation system, Biochim. Biophys. Acta 1102 (1992) 67-75.

[11] B. Korzeniewski, Regulation of ATP supply in mammalian skeletal muscle during resting state --> intensive work transition. Biophys Chem. 83 (2000)19-34.

[12] B. Korzeniewski, J.-P. Mazat, Theoretical studies on the control of oxidative phosphorylation in muscle mitochondria at different energy demands and oxygen concentrations. Acta Biotheoretica 44 (1996) 263-269.

[13] B. Korzeniewski, M. Malgat, T. Letellier, J.-P. Mazat, Effect of binary mitochondria heteroplasmy on respiration and ATP synthesis: implications to mitochondrial diseases, Biochem. J. 357 (2001) 835-842.

[14] R. Bohnensack, Control of energy transformation of mitochondria. Analysis by a quantitative model, Biochim. Biophys. Acta 634 (1981) 203-218.

[15] D.A. Beard, A biophysical model of the mitochondrial respiratory system and oxidative phosphorylation. PloS Computational Biology 1 (2005) 252-264.

[16] S. Helling, M. Hüttemann, R. Ramzan, S. H. Kim, I. Lee, T. Müller, E. Langenfeld, H. E. Meyer, B. Kadenbach, S. Vogt, K. Marcus. Multiple Phosphorylations of Cytochrome $c$ Oxidase and their Functions. Proteomics. 12 (2012) 950-9.

[17] B. Kadenbach, R. Ramzana, L. Wenb, S. Vogt. New extension of the Mitchell Theory for oxidative phosphorylation in mitochondria of living organisms. Biochimica et Biophysica Acta 1800 (2010) 205-212.

[18] V. G. Grivennikova, A. N. Kapustin, A. D. Vinogradov. Catalytic activity of NADHubiquinone oxidoreductase (complex I) in intact mitochondria. Evidence for the slow active/inactive transition. J. Biol Chem, 276(2001) 9038-44,

[19] N. Capitanio, L.L. Palese, G. Capitanio, P.L. Martino, O.M. Richter, B. Ludwig, S. Papa, Allosteric interactions and proton conducting pathways in proton pumping aa(3) oxidases: heme a as a key coupling element. Biochim Biophys Acta 1817 (2012) 558-66.

[20] K.Yugi, M. Tomita, A general computational model of mitochondrial metabolism in a whole organelle scale. Bioinformatics 20 (2004) 1795-1796

[21] N.Berndt, S. Bulik, H.-G. Holzhütter, Kinetic Modeling of the Mitochondrial Energy Metabolism of Neuronal Cells: The Impact of Reduced $\alpha$-Ketoglutarate Dehydrogenase Activities on ATP Production and Generation of Reactive Oxygen Species. International Journal of Cell Biology Volume 2012 (2012), Article ID 757594, 11 pages doi:10.1155/2012/757594

[22] F. Medja, S. Allouche, P. Frachon, C. Jardel, M. Malgat, B. Mousson de Camaret, A. Slama, J. Lunardi, J.P. Mazat, A. Lombès, Development and implementation of standardized respiratory chain spectrophotometric assays for clinical diagnosis, Mitochondrion 9 (2009) 331-339. See also http://mitolab.eu/consensus-protocols-for-clinicaldiagnosis/ 
[23] A. Cornish-Bowden, J.-H.S. Hofmeyr, Enzyme in context: Kinetic characterization of enzymes for systems biology. The Biochemist, (April 2005) 11-14.

[24] C. Chassagnole, B. Raïs, E. Quentin, D.A. Fell, J.-P. Mazat, An integrated study of threonine pathway enzyme kinetics in Escherichia coli. Biochem. J. 356, (2001) 415-423.

[25] J. M. Rohwer, Kinetic modelling of plant metabolic pathways Journal of Experimental Botany, 63 (2012) 2275-2292.

[26] I. H. Segel. Rapid equilibrium bireactant systems. In I. H. Segel, editor, Enzyme Kinetics. Behavior and Analysis of Rapid Equilibrium and Steady-State Enzyme Systems. John Wiley \& Sons, New York, wiley classics library edition, 1993. ISBN 978-0-471-30309-1.

[27] W. Liebermeister, E. Klipp, Bringing metabolic network to life: convenience rate law and thermodynamic constraints. Theor. Biol. and Med. Mod., 3 (2006) 41.

[28] S. Ransac, M. Heiske, J.-P. Mazat : From in silico to in spectro Kinetics of Respiratory Complex I. Biochim. Biophys. Acta 1817 (2012) 1958-1969.

[29] ] A.L. Smith, Preparation, properties, and conditions for assay of mitochondria: Slaughterhouse material, small-scale. Methods in Enzymology 10 (1967) 81-86.

[30] P. Mendes, D. Kell, Non-linear optimization of biochemical pathways: applications to metabolic engineering and parameter estimation, Bioinformatics 14 (1998) 869-883.

[31] V. Henri, Théorie générale de l'action de quelques diastases. Compte Rend. Acad. Sci. Paris 135 (1902) 916-919.

[32] L. Michaelis, M.L. Menten, Die Kinetic der Invertinwirkung. Biochem. Z. 49 (1913) 333-369.

[33] G.E. Briggs J.B.S. Haldane, A note on the kinetics of enzyme action. Biochem. J. 19 (1925) 338-339.

[34] U. Deichmann,S. Schuster,J.-P. Mazat,A. Cornish-Bowden, Commemorating the 1913 Michaelis-Menten paper Die Kinetik der Invertinwirkung: three perspectives. FEBS J. 281 (2014) 435-63. 
[35] W. Liebermeister, J. Uhlendorf, E. Klipp, Modular rate laws for enzymatic reactions: thermodynamics, elasticities and implementation Bioinformatics. 26 (2010) 1528-34.

[36] C.S. Pillay, J.-H. S. Hofmeyr, B.G. Olivier, J.L. Snoep, J.M. Rohwer, Enzymes or redox couples? The kinetics of thioredoxin and glutaredoxin reactions in a systems biology context. Biochem. J. 417 (2009) 269-275.

[37] R. Fato, E. Estornell, S. Di Bernardo, F. Pallotti, G. Parenti Castelli, G. Lenaz, Steady-state kinetics of the reduction of coenzyme $Q$ analogs by complex I (NADH:ubiquinone oxidoreductase) in bovine heart mitochondria and submitochondrial particles, Biochemistry 35 (1996) 2705-2716.

[38] Y. Nakashima, K. Shinzawa-Itoh, K. Watanabe, K. Naoki, N. Hano, S. Yoshikawa, Steadystate kinetics of NADH:coenzyme Q oxidoreductase isolated from bovine heart mitochondria, J. Bioenerg. Biomembr. 34 (2002) 11-19.

[39] N. Hano, Y. Nakashima, K. Shinzawa-Itoh, S. Yoshikawa, Effect of the side chain structure of coenzyme Q on the steady state kinetics of bovine heart NADH: coenzyme Q oxidoreductase, J. Bioenerg. Biomembr. 35 (2003) 257-265.

[40] D.T. Gillespie, Exact stochastic simulation of coupled chemical reactions, J. Phys. Chem. 81 (1977) 2340-2361.

[41] S. Sherwood, J. Hirst, Investigation of the mechanism of proton translocation by NADH:ubiquinone oxidoreductase (complex I) from bovine heart mitochondria: does the enzyme operate by a Q-cycle mechanism? Biochem. J. 400 (2006) 541-550.

[42] M. Ohshima, H. Miyoshi, K. Sakamoto, K. Takegami, J. Iwata, K. Kuwabara, H. Iwamura, T. Yagi, Characterisation of the ubiquinone reduction site of mitochondrial complex I using bulky synthetic ubiquinones. Biochemistry 37 (1998) 6436-644.

[43] A.D. Vinogradov, Kinetics, Control, and Mechanism of Ubiquinone Reduction by the Mammalian Respiratory Chain-Linked NADH-Ubiquinone Reductase. J. Bioenerg. Biomembr. 25 (1993) 367-375.

[44] Y. Nakashima, K. Shinzawa-Itoh, K. Watanabe, K. Naoki, N. Hano, S. Yoshikawa, The second coenzyme Q1 binding site of bovine heart NADH: coenzyme Q oxidoreductase, J. Bioenerg. Biomembr. 34 (2002) 89-94.

[45] A.D. Vinogradov, Catalytic properties of the mitochondrial NADH-ubiquinone oxidoreductase (complex I) and the pseudo-reversible active/inactive enzyme transition, Biochim. Biophys. Acta 1364 (1998) 169-185.

[46] G. Lenaz, P. Pasquali, E. Bertoli, G. Parenti-Castelli. The inhibition of NADH oxidase by the lower homologs of coenzyme Q. Arch Biochem Biophys, 169(1975) 217-26. 
[47] T. Ohnishi, J.E. Johnson Jr., T. Yano, R. Lobrutto, W.R. Widger, Thermodynamic and EPR studies of slowly relaxing ubisemiquinone species in the isolated bovine heart complex I, FEBS Lett. 579 (2005) 500-506.

[48] S. Magnitsky, L. Toulokhonova, T. Yano, V.D. Sled, C. Hägerhäll, V.G. Grivennikova, D.S. Burbaev, A.D. Vinogradov, T. Ohnishi, EPR characterization of ubisemiquinones and iron-sulfur cluster N2, central components of the energy coupling in the NADH-ubiquinone oxidoreductase (complex I) in situ, J. Bioenerg. Biomembr. 34 (2002) 193-208.

[49] R. Baradaran, J.M. Berrisford, G.S. Minhas, L.A. Sazanov, Crystal structure of the entire respiratory complex I. Nature 494 (2013) 443-48

[50] C. Hunte, V. Zickermann, U. Brandt, Functional modules and structural basis of coformational coupling in mitochondrial complex I, Science 329 (2010) 448-451.

[51] Heiske M. PhD thesis : http://www.bu.u-bordeaux2.fr/babordplus.php?special=these 


\section{Legend to the Figures.}

Figure 1: Mechanisms associated to the rate equations. MAL means Mass Action Law. In ER-HMM (Extended Reversible-Henri-Michaelis-Menten) and in CK (Convenient Kinetics) mechanisms, ENH means E-NADH, ENHQ: E-NADH-Q, ENHQH 2 : E-NADH-QH 2 , ENQ: E-NAD-Q, ENQH 2 : E-NAD-QH ${ }_{2}, \mathrm{EQH}_{2}: \mathrm{E}_{2} \mathrm{QH}_{2}$ and EN: E-NAD. In EMA mechanism 1, E* symbolizes an Enzyme-Substrates-Products complex. In mechanism 2, $\mathrm{E}_{\mathrm{A}}$ and $\mathrm{E}_{\mathrm{B}}$ represent two enzyme conformations able to bind respectively the substrates only and the products only. The derivations of EMA equation are detailed in Supplementary Materials 8.

Figure 2: Comparison of different rate equation to fit part of the experimental results. The kinetic parameters are determined by minimizing the root mean square deviation (RMSD) calculated between all experimental data and the corresponding theoretical points evaluated with the equation under study (see table 1) as explained in the Materials and Methods section. Note that the fitting procedure is performed on all experimental results represented in Supplementary Materials, but that only a small part of them is represented in this comparison for clarity. The experimental points are in black and the fitting curves in red.

(a) and (b): the experimental points are fitted by the NET equation.

(c) and (d): the experimental points are fitted by the MAL equation.

(e) and (f): the experimental points are fitted by the ER-HMM equation.

$(\mathrm{g})$ and $(\mathrm{h})$ : the experimental points are fitted by EMA equation.

The parameters for tracing the theoretical curves are listed in table 1.

Other parameters. NET: $\mathrm{k}_{\mathrm{CI}}=6.6$ nmoles $/ \mathrm{min} / \mathrm{mg}$; MAL: $\mathrm{k}_{\mathrm{f}}=3.16$ nmoles. $(\mu \mathrm{M})^{-2} \cdot \mathrm{min}^{-1} \cdot \mathrm{mg}^{-1}$; $\mathrm{k}_{\mathrm{b}}=2.0410^{-11}$ nmoles. $(\mu \mathrm{M})^{-2} \cdot \mathrm{min}^{-1} \cdot \mathrm{mg}^{-1}$.

NADH variable : $(\mathrm{O}) \mathrm{Q}=1.1 \mu \mathrm{M} ;(\Delta) \mathrm{Q}=4.4 \mu \mathrm{M} ;(\diamond) \mathrm{Q}=10.9 \mu \mathrm{M} ;(\square) \mathrm{Q}=32.7 \mu \mathrm{M}$ Q variable: $(\mathrm{X}) \mathrm{NADH}=0.95 \mu \mathrm{M} ;(\mathrm{O}) \mathrm{NADH}=3.8 \mu \mathrm{M} ;(\Delta) \mathrm{NADH}=6.65 \mu \mathrm{M} ;(\diamond) \mathrm{NADH}$ $=14.25 \mu \mathrm{M} ;(\square) \mathrm{NADH}=28.5 \mu \mathrm{M}$.

Figure 3 Products inhibition.

Figure 2-a: NAD inhibition with $\mathrm{NADH}=38 \mu \mathrm{M}$ and $\mathrm{Q}=69 \mu \mathrm{M}$ (See also figures $\mathrm{z}$ to ac in the Supplementary Materials. This figure corresponds to figure ac with ER-HMM equation). Figure 2-b: $\mathrm{QH}_{2}$ inhibition with $\mathrm{NADH}=28.5 \mu \mathrm{M}$ and $\mathrm{Q}=54.5 \mu \mathrm{M}$

(See also figures $\mathrm{v}$ and $\mathrm{w}$ in the Supplementary Materials. This figure corresponds to figure $\mathrm{w}$ with ER-HMM equation).

The fitting curve is represented by a continuous red line.

\section{Figure 4: Complex I inhibition by the substrate Q.}

The curves are drawn according to the ER-HMM equation (eq.9) with the parameters of table 2 in the absence of $\mathrm{Q}$ substrate inhibition (- - ) and with the term Is $=1+\mathrm{Q} / \mathrm{K}_{\mathrm{I}}$ (red large line) with the parameters of table 3 , second row $\left(\mathrm{k}_{\mathrm{f}}=2194, \mathrm{~K}_{\mathrm{NADH}}=4.5 \mu \mathrm{M}, \mathrm{K}_{\mathrm{Q}}=14.7 \mu \mathrm{M}, \mathrm{K}_{\mathrm{NAD}}\right.$ $=743 \mu \mathrm{M}, \mathrm{K}_{\mathrm{QH} 2}=7.5 \mu \mathrm{M}$ and $\left.\mathrm{K}_{\mathrm{I}}=576 \mu \mathrm{M}\right)$ or with the term Inc $=1 /\left(1+\mathrm{Q} / \mathrm{K}_{\mathrm{I}}\right)($ thin black line overlapped by the red one) with the parameters of table 3 , third row $\left(\mathrm{k}_{\mathrm{f}}=2254, \mathrm{~K}_{\mathrm{NADH}}=\right.$ $4.5 \mu \mathrm{M}, \mathrm{K}_{\mathrm{Q}}=15.1 \mu \mathrm{M}, \mathrm{K}_{\mathrm{NAD}}=743 \mu \mathrm{M}, \mathrm{K}_{\mathrm{QH} 2}=7.6 \mu \mathrm{M}$ and $\left.\mathrm{K}_{\mathrm{I}}=561 \mu \mathrm{M}\right)$.

The black diamonds correspond to the experimental points at (a) $\mathrm{NADH}=28.5 \mu \mathrm{M}$, (b) $\mathrm{NADH}=47.5 \mu \mathrm{M}$. (c) depicts the rate as a function of $\mathrm{QH}_{2}$ with $\mathrm{NADH}=28.5 \mu \mathrm{M}$ and $\mathrm{Q}=$ $45.5 \mu \mathrm{M}$ with steric inhibition ( - ) or non competitive inhibition ( - superimposed with the previous one - ) or without any inhibition ( $--=)$.

The fit of the other inhibitory function are very similar (not shown). See also figures S6 and S7 in Supplementary Materials. 
Table 1: The different equations discussed in this paper.

\begin{tabular}{|c|c|c|}
\hline Name and abbreviation & EQUATION & Equilibrium constraints \\
\hline $\begin{array}{l}\text { Extended Reversible } \\
\text { Henri-Michaelis-Menten } \\
\text { (ER-HMM equation) }\end{array}$ & $v=E_{t} \frac{k_{f} \cdot \frac{N A D H}{K_{N A D H}} \cdot \frac{Q}{K_{Q}}-k_{b} \cdot \frac{N A D}{K_{N A D}} \cdot \frac{Q H_{2}}{K_{Q H_{2}}}}{\left(1+\frac{N A D H}{K_{N A D H}}+\frac{N A D}{K_{N A D}}\right)\left(1+\frac{Q}{K_{Q}}+\frac{Q H_{2}}{K_{Q H_{2}}}\right)}$ & $K_{e q}=\frac{k_{f}}{k_{b}} \cdot \frac{K_{N A D}}{K_{N A D H}} \cdot \frac{K_{Q H_{2}}}{K_{Q}}$ \\
\hline $\begin{array}{l}\text { Convenience Kinetics } \\
\text { (CK equation) }\end{array}$ & $v=E_{t} \frac{k_{f} \cdot \frac{N A D H}{K_{N A D H}} \cdot \frac{Q}{K_{Q}}-k_{b} \cdot \frac{N A D}{K_{N A D}} \cdot \frac{Q H_{2}}{K_{Q H_{2}}}}{\left(1+\frac{N A D H}{K_{N A D H}}\right)\left(1+\frac{Q}{K_{Q}}\right)+\left(1+\frac{N A D}{K_{N A D}}\right)\left(1+\frac{Q H_{2}}{K_{Q H_{2}}}\right)-1}$ & $K_{e q}=\frac{k_{f}}{k_{b}} \cdot \frac{K_{N A D}}{K_{N A D H}} \cdot \frac{K_{Q H_{2}}}{K_{Q}}$ \\
\hline $\begin{array}{l}\text { Extended Mass Action } \\
\text { Equation } \\
\text { (EMA equation) }\end{array}$ & $v=E_{t} \frac{k_{f} \cdot \frac{N A D H \cdot Q}{C_{S}}-k_{b} \cdot \frac{N A D \cdot Q H_{2}}{C_{P}}}{1+\frac{N A D H \cdot Q}{C_{S}}+\frac{N A D \cdot Q H_{2}}{C_{P}}}$ & $K_{e q}=\frac{k_{f}}{k_{b}} \cdot \frac{C_{P}}{C_{S}}$ \\
\hline $\begin{array}{l}\text { Ordered mechanism } \\
\quad \text { (Inversed) } * \\
\text { OM(I) equation }\end{array}$ & $v_{O M}=E_{t} \cdot \frac{k_{f} \cdot \frac{N A D H}{K_{N A D H}} \cdot \frac{Q}{K_{Q}}-k_{b} \cdot \frac{N A D}{K_{N A D}} \cdot \frac{Q H_{2}}{K_{Q H 2}}}{\left[\begin{array}{l}1+\frac{N A D H}{K_{N A D H}}+\frac{Q}{K_{Q}}+\frac{N A D}{K_{N A D}}+\frac{Q H_{2}}{K_{Q H 2}}+\frac{N A D H}{K_{N A D H}} \cdot \frac{Q}{K_{Q}}+\frac{N A D}{K_{N A D} D} \frac{Q H_{2}}{K_{Q H 2}}+ \\
\frac{N A D H}{K_{N A D H}} \cdot \frac{Q H_{2}}{K_{Q H 2}}+\frac{Q}{K_{Q}} \cdot \frac{N A D}{K_{N A D}}+\frac{N A D H}{K_{N A D H}} \cdot \frac{Q}{K_{Q}} \cdot \frac{N A D}{K_{N A D}}+\frac{Q}{K_{Q}} \cdot \frac{N A D}{K_{\text {NAD }}} \cdot \frac{Q H_{2}}{K_{Q H 2}}\end{array}\right]}$ & $K_{e q}=\frac{k_{f}}{k_{b}} \cdot \frac{K_{N A D}}{K_{N A D H}} \cdot \frac{K_{Q H_{2}}}{K_{Q}}$ \\
\hline $\begin{array}{l}\text { Ping-Pong Mechanism } \\
\text { PPM equation }\end{array}$ & $v_{P P M}=E_{t} \cdot \frac{k_{f} \cdot \frac{N A D H}{K_{N A D H}} \cdot \frac{Q}{K_{Q}}-k_{b} \cdot \frac{N A D}{K_{N A D}} \cdot \frac{Q H_{2}}{K_{Q H 2}}}{\left[\begin{array}{l}\frac{N A D H}{K_{N A D H}}+\frac{Q}{K_{Q}}+\frac{N A D}{K_{N A D}}+\frac{Q H_{2}}{K_{\text {OH }}}+\frac{N A D H}{K_{N A D H}} \cdot \frac{Q}{K_{Q}}+ \\
\frac{N A D}{K_{N A D}} \cdot \frac{Q H_{2}}{K_{Q H 2}}+\frac{N A D H}{K_{N A D H}} \cdot \frac{N A D}{K_{N A D}}+\frac{Q}{K_{Q}} \cdot \frac{Q H_{2}}{K_{Q H 2}}\end{array}\right]}$ & $K_{e q}=\frac{k_{c a t f}}{k_{c a t b}} \cdot \frac{K_{N A D^{+}} \cdot K_{Q H_{2}}}{K_{N A D H} \cdot K_{Q}}$ \\
\hline
\end{tabular}


Table 2: Fits of the experimental points by the different saturable equations of table 1. The kinetic parameters are identified by minimizing the root mean square deviation (RMSD) calculated between all experimental data and the corresponding theoretical points evaluated with the equation under study as explained in the Materials and Methods section (see Supplementary Materials for the figures of all fits). The $\mathrm{k}_{\mathrm{b}}$ value has been calculated using the Haldane relationship with $\mathrm{K}_{\mathrm{eq}}=1.5410^{11}$ corresponding to $\Delta \mathrm{G}_{\mathrm{o}}{ }_{\mathrm{o}}(\mathrm{pH} 7.5 ; \mathrm{T}=310 \mathrm{~K})=-66.4$ $\mathrm{kJ} / \mathrm{mole}$

\begin{tabular}{|c|c|c|c|c|c|c|c|}
\hline Equation & RMSD & $\underset{[\mathrm{nmol}(\mathrm{NAD}}{\mathbf{k}_{\mathbf{f}}}$ & $\begin{array}{c}\mathbf{k}_{\mathbf{b}} \\
\left.\min ^{-1} \mathrm{mg}^{-1}\right]\end{array}$ & $\begin{array}{c}\mathbf{K}_{\text {NADH }} \\
{[\mu \mathrm{M}]}\end{array}$ & $\begin{array}{c}\mathbf{K}_{\mathbf{Q}} \\
{[\mu \mathrm{M}]}\end{array}$ & $\begin{array}{c}\mathbf{K}_{\mathrm{NAD}} \\
{[\mu \mathrm{M}]}\end{array}$ & $\begin{array}{c}\mathbf{K}_{\mathbf{Q H 2}} \\
{[\mu \mathrm{M}]}\end{array}$ \\
\hline PPM & 162 & 1910 & $1.3 \cdot 10^{-6}$ & 6.1 & 13.1 & 2064 & 4.0 \\
\hline $\begin{array}{l}\text { OM } \\
\text { (NQNQ) }\end{array}$ & 161 & 1802 & $1.0 \cdot 10^{-5}$ & 4.4 & 9.9 & 13931 & 2.7 \\
\hline $\begin{array}{l}\text { OM } \\
\text { (QNQN) }\end{array}$ & 162 & 1797 & $5.0 \cdot 10^{-6}$ & 4.3 & 9.8 & 774 & 23.6 \\
\hline $\begin{array}{l}\text { OM } \\
\text { (QNNQ) }\end{array}$ & 164 & 1801 & $1.9 \cdot 10^{-5}$ & 4.4 & 9.7 & 13087 & 5.3 \\
\hline $\begin{array}{l}\text { OM } \\
(\mathrm{NQQN})\end{array}$ & 168 & 1793 & $9.2 \cdot 10^{-6}$ & 4.3 & 9.8 & 1666 & 20.1 \\
\hline ER-HMM & 164 & 1791 & $1.1 \cdot 10^{-6}$ & 4.3 & 9.7 & 780 & 5.3 \\
\hline CK & 167 & 1773 & $1.5 \cdot 10^{-8}$ & 4.2 & 9.5 & 88 & 0.6 \\
\hline EMA & 244 & 1333 & $1.8 \cdot 10^{-10}$ & \multicolumn{2}{|c|}{$\mathrm{C}_{\mathrm{S}}=166 \mu \mathrm{M}^{2}$} & \multicolumn{2}{|c|}{$\mathrm{C}_{\mathrm{P}}=3.4 \mu \mathrm{M}^{2}$} \\
\hline
\end{tabular}

Table 3: Competitive/steric and non-competitive inhibition of complex I by quinones. The table lists the results of fitting all the experimental data with an inhibition term in the ERHMM equation (eq. (10)) as explained in the text. The fitting method is the same as in table 2. When there is only one inhibitory constant, it is called $\mathrm{K}_{\mathrm{i}}$ and listed in $\mathrm{K}_{\mathrm{i} 1}$ column. The $\mathrm{k}_{\mathrm{b}}$ value has been calculated using the Haldane relationship as in table 2.

\begin{tabular}{|c|c|c|c|c|c|c|c|c|c|}
\hline Inhibition & RMSD & $\begin{array}{c}\mathbf{k}_{\mathrm{f}} \\
1 \mathrm{NAD}\end{array}$ & $\left.\begin{array}{c}\mathbf{k}_{\mathbf{b}} \\
\left.\mathrm{min}^{-1} \mathrm{mg}^{-1}\right]\end{array}\right]$ & $\begin{array}{c}\mathbf{K}_{\mathrm{NADH}} \\
{[\mu \mathrm{M}]}\end{array}$ & $\begin{array}{c}\mathbf{K}_{\mathbf{Q}} \\
{[\mu \mathrm{M}]}\end{array}$ & $\begin{array}{l}\mathbf{K}_{\mathrm{NAD}} \\
{[\mu \mathrm{M}]}\end{array}$ & $\begin{array}{l}\mathbf{K}_{\mathrm{QH} 2} \\
{[\mu \mathrm{M}]}\end{array}$ & $\begin{array}{c}\mathbf{K i}_{1} \\
{[\mu \mathrm{M}]}\end{array}$ & $\begin{array}{c}\mathbf{K i}_{2} \\
{[\mu \mathrm{M}]}\end{array}$ \\
\hline$I_{s}=1+\frac{Q}{K i_{1}}+\frac{Q H_{2}}{K i_{2}}$ & 151 & 2185 & $7.42 \cdot 10^{-6}$ & 4.5 & 14.7 & 740 & 46.9 & 598 & 25.4 \\
\hline$I_{s}=1+\frac{Q_{t o t}}{K i}$ & 153 & 2194 & $1.2010^{-6}$ & 4.5 & 14.7 & 743 & 7.5 & 576 & \\
\hline$I_{n c}=\frac{1}{1+\frac{Q_{t o t}}{U i}}$ & 153 & 2254 & $1.21 \cdot 10^{-6}$ & 4.5 & 15.1 & 743 & 7.6 & 561 & \\
\hline$I_{n c}=\frac{1^{\mathbf{K}}}{1+\frac{Q}{K i}}$ & 154 & 2244 & $1.07 \cdot 10^{-6}$ & 4.5 & 15.0 & 744 & 6.7 & 575 & \\
\hline$I_{n c}=\left(\frac{1}{1+\frac{Q_{t o t}}{K i}}\right)^{2}$ & 153 & 2209 & $1.18 \cdot 10^{-6}$ & 4.5 & 14.7 & 740 & 7.4 & 1319 & \\
\hline$I_{n c}=\left(\frac{1}{1+\frac{Q}{K i}}\right)^{2}$ & 154 & 2200 & $1.07 \cdot 10^{-6}$ & 4.5 & 14.5 & 741 & 6.6 & 1351 & \\
\hline$I_{n c}=\frac{1}{1+\frac{Q}{K i}+\frac{Q H_{2}}{K i_{2}}}$ & 152 & 2236 & $1.04 \cdot 10^{-5}$ & 4.5 & 15.0 & 741 & 65.6 & 589 & 29.8 \\
\hline$I_{n c}=\left(\frac{1}{1+\frac{Q}{K i}+\frac{Q H_{2}}{K i_{2}}}\right)$ & 151 & 2198 & $2.83 \cdot 10^{-5}$ & 4.5 & 14.6 & 737 & 177 & 1367 & 56.8 \\
\hline
\end{tabular}


Figure 1

(NAME


Figure 2

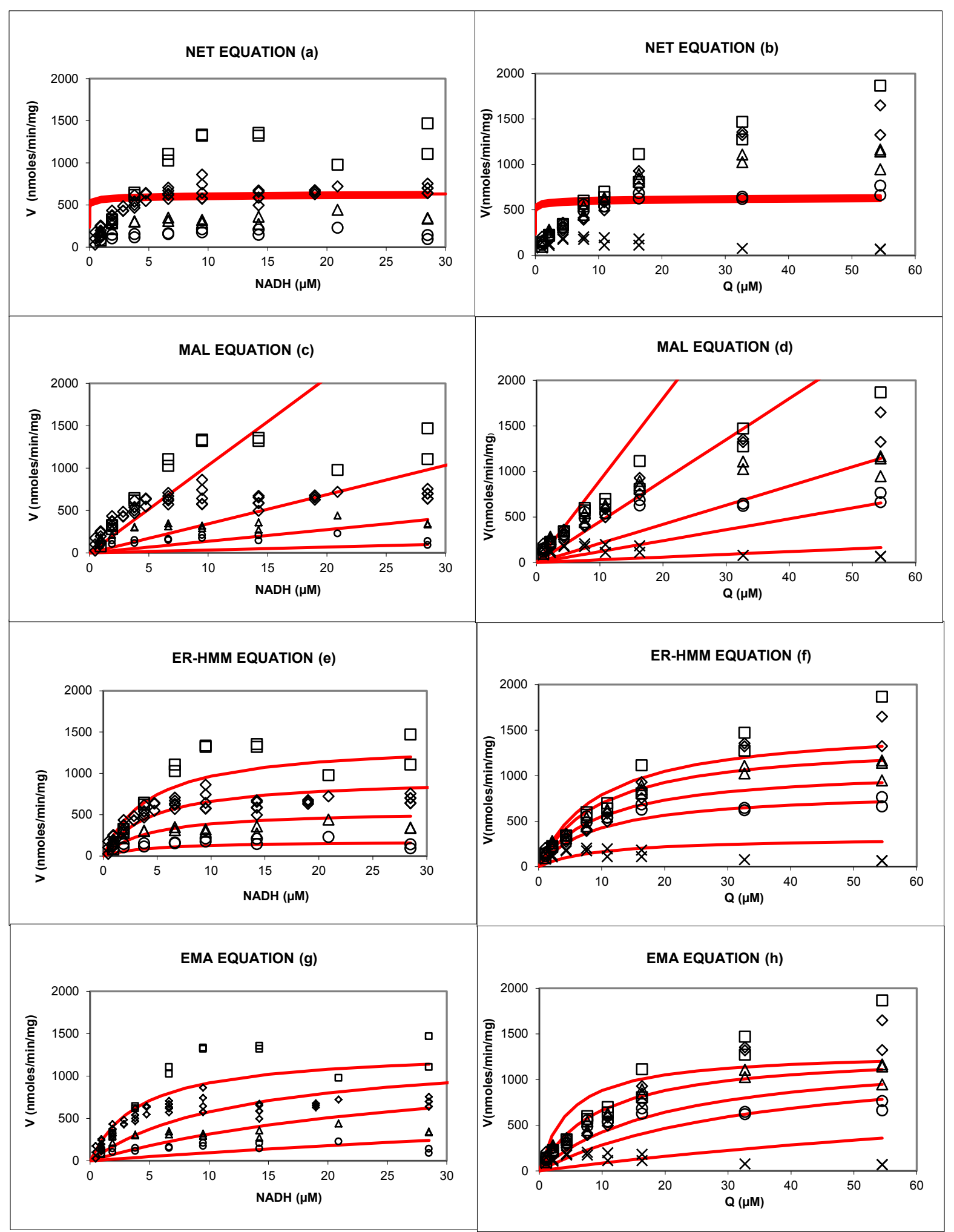


Figure 3
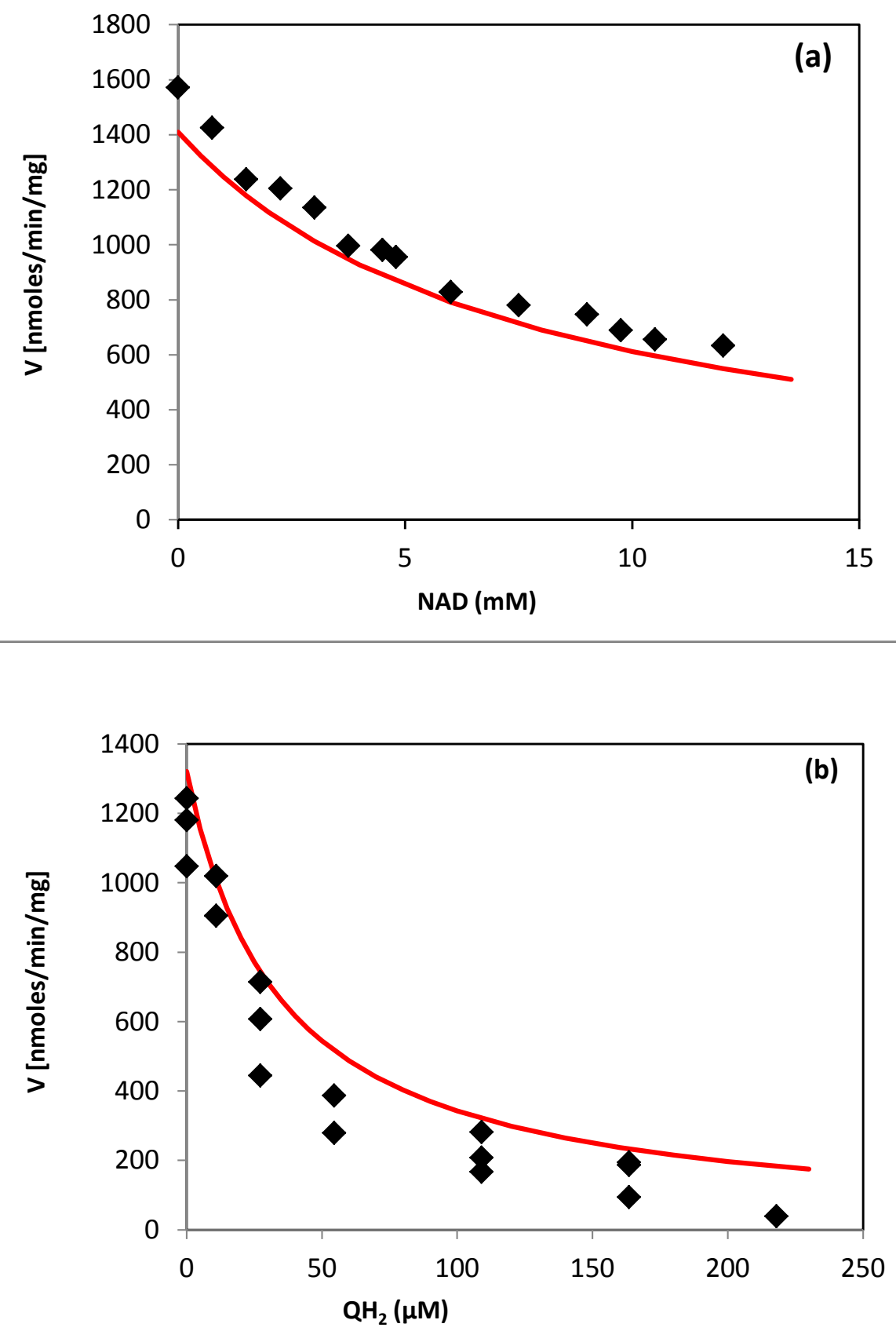
Figure 4

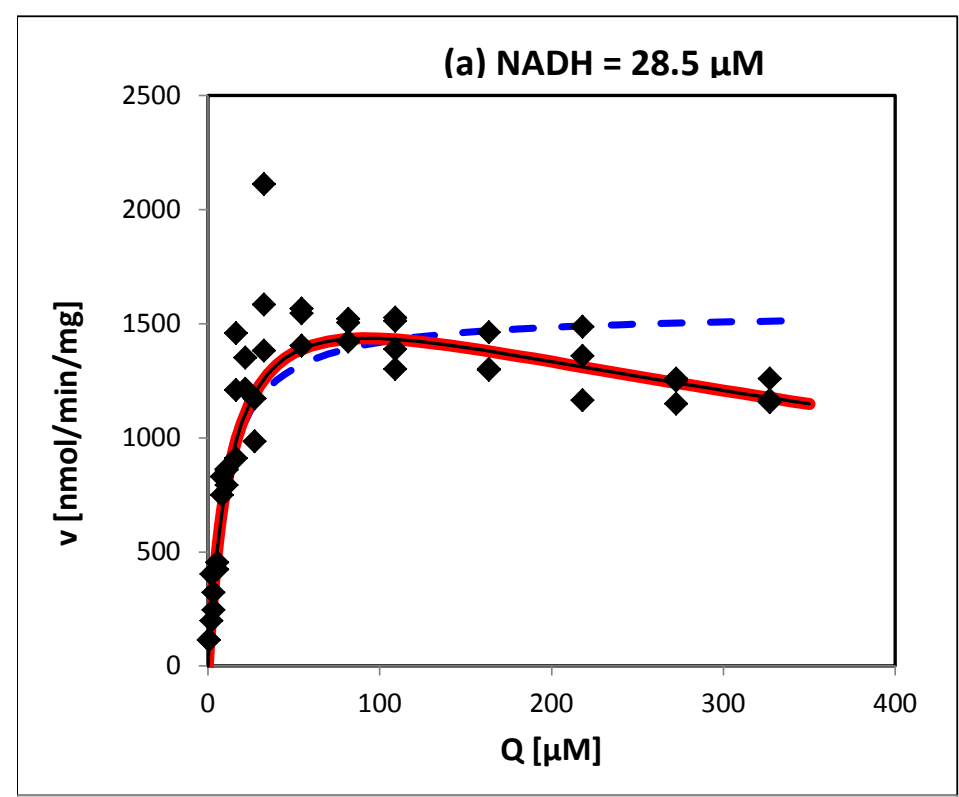

(b) $\mathrm{NADH}=47.5 \mu \mathrm{M}$

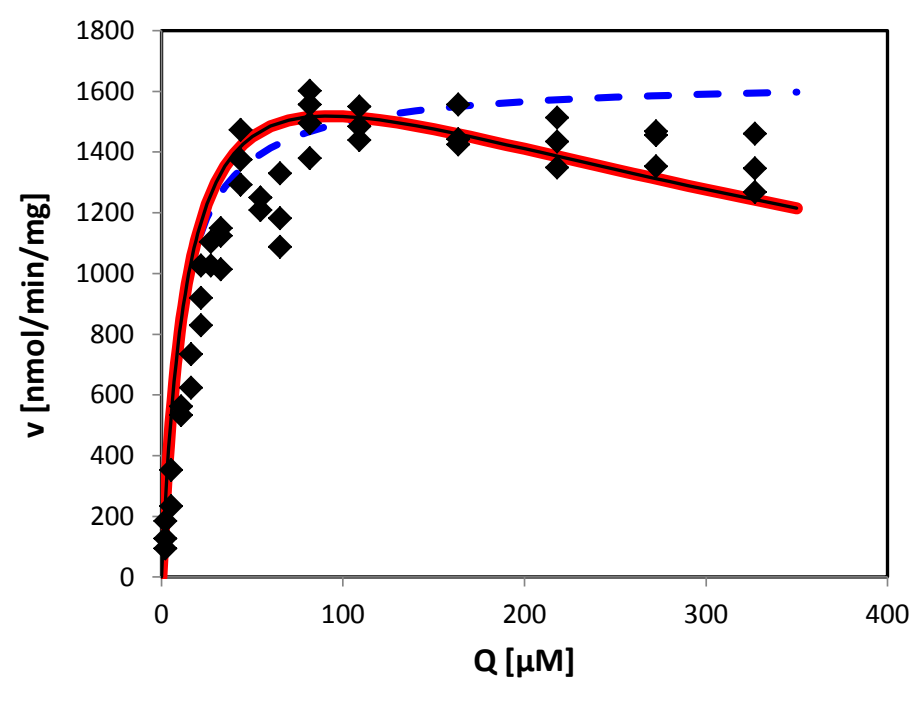

(c) $\mathrm{QH}_{2}$ variation,

$\mathrm{NADH}=28.5 \mu \mathrm{M}, \mathrm{Q}=45.5 \mu \mathrm{M}$

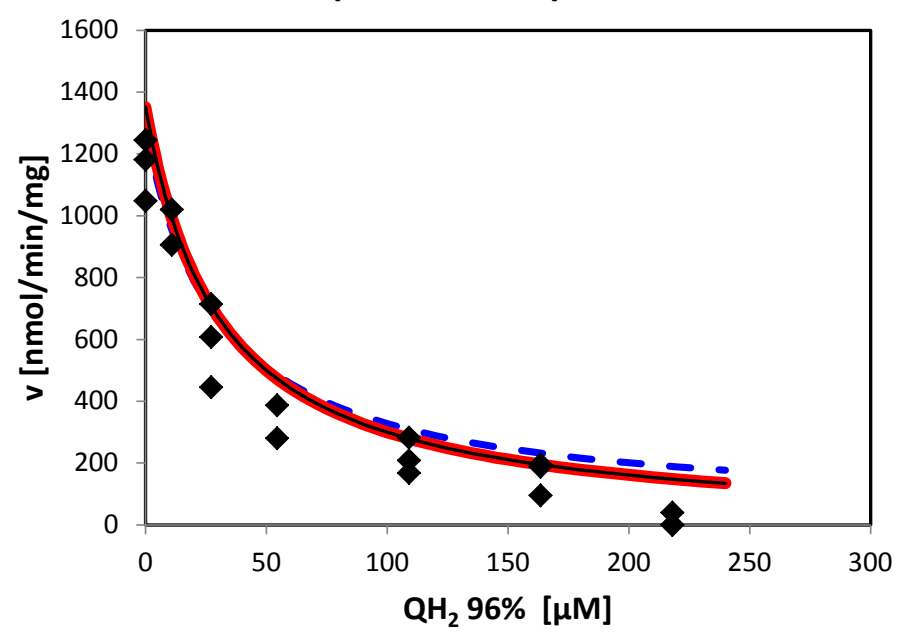




\section{Supplementary Material S1 : Non-saturable rate equations}

\section{I-1 Near Equilibrium Thermodynamics (NET) Equation}

In the near equilibrium approach the reaction velocity is assumed to be proportional to the Gibbs energy.

$v_{C I}=-k_{C I} \Delta G_{C I}$

with :

$$
\Delta G_{C I}=\Delta G_{o, C I}^{\prime}+R T \cdot \operatorname{Ln}\left[\frac{[N A D] \cdot\left[Q H_{2}\right]}{[N A D H] \cdot[Q]}\right] \quad \text { (7) where }[\mathrm{X}] \text { denotes the concentration of }
$$

metabolite X.

This type of equation was proposed by Westerhoff [7] and used by Korzeniewski et al. [10] for instance to model complexes I and III of respiratory chain.

The major advantage of this equation is its simplicity, since there is only one parameter to determine, besides the standard Gibbs energy which is known for the respiratory chain complexes. Furthermore one can be certain that the flux vanishes at equilibrium (for $\Delta G_{C I}=$ 0 ). Another point is that the equation can deal with any stoichiometric factor, also non-integer ones, which can be advantageous e.g. for the more complex stoichiometries of complex III and IV of the respiratory chain. Finally, in this equation, it is easy to take into account the proton-motive force $\Delta \mu_{\mathrm{H}}{ }^{+}$as an extra term added to $\Delta \mathrm{G}_{\mathrm{CI}}$ of the chemical reaction [10].

The fact that the concentrations appear as a logarithmical term limits the increase of velocity with increasing substrate concentration. This does not correspond to a typical saturation behaviour of an enzyme but the non-linear increase of velocity somehow approaches this property, as we can see in Fig.2 a and b. However, the logarithmic expression of the concentrations leads to a very high impact of very small substrate concentrations (near zero) on the velocity, i.e. at low substrate concentration, a small change results in a great velocity change, and on the other side at higher concentrations, the velocity hardly changes, giving nearly overlapping curves when the other substrate is varied. As shown on Fig. $2 a$ and b, the initial velocities measured experimentally in the absence of products cannot be fitted correctly with this equation. It is predictable because in absence of products and at $\Delta \mu_{\mathrm{H}}{ }^{+}=0$ with a $\Delta \mathrm{G}^{\circ}{ }_{\mathrm{CI}}=-66.4 \mathrm{~kJ} / \mathrm{mol}$ this reaction is always very far from equilibrium.

\section{I-2 The Mass Action Law equation.}


The MAL equation applied to the enzymatic reaction (1) reads:

$v_{C I}=k_{f}[N A D H] .[Q]-k_{b}[N A D] .\left[Q H_{2}\right]$

or introducing $\mathrm{K}_{\mathrm{eq}}: v_{C I}=k_{b}\left(K_{e q}[N A D H] .[Q]-[N A D] .\left[Q H_{2}\right]\right)$

This type of equation was largely used for modelling the respiratory chain complexes (e.g. [14-15]). As previously, introducing the $\mathrm{K}_{\mathrm{eq}}=\mathrm{k}_{\mathrm{f}} / \mathrm{k}_{\mathrm{b}}$ allows one to be sure that $\mathrm{v}_{\mathrm{CI}}$ vanishes at equilibrium. Additional terms in the form of $\exp \left[-\mathrm{n}_{\mathrm{I}} \mathrm{RT} \ln \left(\left[\mathrm{H}^{+}{ }_{\text {in }}\right] /\left[\mathrm{H}^{+}{ }_{\text {out }}\right]\right)\right]$ can be added (as in [15]) to take the proton-motive force into account where $\mathrm{n}_{\mathrm{I}}$ is the number of protons expelled by complex I. The equation has the same amount of parameters as the NET equation and is also not limited to particular stoichiometric factors.

A rather cumbersome drawback of this equation is that the velocity increases proportionally to the substrate concentrations. This does not correspond to the typical saturation behaviour of enzymes (see Fig.2 $\mathrm{c}$ and d). This is the main reason for which this equation fails to fit the initial velocities measured for different substrate concentrations.

We conclude that these equations, MAL as well as the NET equation cannot be used for an accurate description of respiratory complexes kinetics when there is a possible variation in substrates and products concentrations. It is necessary to introduce a saturation term in the rate equations. 
Table 1: The different equations discussed in this paper.

\begin{tabular}{|c|c|c|}
\hline Name and abbreviation & EQUATION & Equilibrium constraints \\
\hline $\begin{array}{l}\text { Near Equilibrium } \\
\text { Thermodynamics } \\
\text { (NET equation) }\end{array}$ & $v_{C I}=-k_{C I} \Delta G_{C I}$ & $\Delta G_{C I}=\Delta G_{o, C I}^{\prime}+R T \cdot \ln \left[\frac{\left[N A D^{+}\right] \cdot\left[Q H_{2}\right]}{[N A D H] \cdot[Q]}\right.$ \\
\hline $\begin{array}{l}\text { Mass Action Law } \\
\text { (MAL equation) }\end{array}$ & $\begin{array}{c}\mathrm{NADH}+\mathrm{H}^{+}+\mathrm{Q} \underset{\mathrm{k}_{\mathrm{b}}}{\stackrel{\mathrm{k}_{\mathrm{f}}}{\rightleftarrows}} \mathrm{NAD}^{+}+\mathrm{QH}_{2} \\
v_{C I}=k_{f}[N A D H] .[Q]-k_{b}[N A D] .\left[Q H_{2}\right]\end{array}$ & $K_{e q}=k_{f} / k_{b}$ \\
\hline $\begin{array}{l}\text { Extended Reversible } \\
\text { Henri-Michaelis-Menten } \\
\text { (ER-HMM equation) }\end{array}$ & $v=E_{t} \frac{k_{f} \cdot \frac{N A D H}{K_{N A D H}} \cdot \frac{Q}{K_{Q}}-k_{b} \cdot \frac{N A D}{K_{N A D}} \cdot \frac{Q H_{2}}{K_{Q H_{2}}}}{\left(1+\frac{N A D H}{K_{N A D H}}+\frac{N A D}{K_{N A D}}\right)\left(1+\frac{Q}{K_{Q}}+\frac{Q H_{2}}{K_{Q H_{2}}}\right)}$ & $K_{e q}=\frac{k_{f}}{k_{b}} \cdot \frac{K_{N A D}}{K_{N A D H}} \cdot \frac{K_{Q H_{2}}}{K_{Q}}$ \\
\hline $\begin{array}{l}\text { Convenience Kinetics } \\
\text { (CK equation) }\end{array}$ & $v=E_{t} \frac{k_{f} \cdot \frac{N A D H}{K_{N A D H}} \cdot \frac{Q}{K_{Q}}-k_{b} \cdot \frac{N A D}{K_{N A D}} \cdot \frac{Q H_{2}}{K_{Q H_{2}}}}{\left(1+\frac{N A D H}{K_{N A D H}}\right)\left(1+\frac{Q}{K_{Q}}\right)+\left(1+\frac{N A D}{K_{N A D}}\right)\left(1+\frac{Q H_{2}}{K_{Q H_{2}}}\right)-1}$ & $K_{e q}=\frac{k_{f}}{k_{b}} \cdot \frac{K_{N A D}}{K_{N A D H}} \cdot \frac{K_{Q H_{2}}}{K_{Q}}$ \\
\hline $\begin{array}{l}\text { Extended Mass Action } \\
\text { Equation } \\
\text { (EMA equation) }\end{array}$ & $v=E_{t} \frac{k_{f} \cdot \frac{N A D H \cdot Q}{C_{S}}-k_{b} \cdot \frac{N A D \cdot Q H_{2}}{C_{P}}}{1+\frac{N A D H \cdot Q}{C_{S}}+\frac{N A D \cdot Q H_{2}}{C_{P}}}$ & $K_{e q}=\frac{k_{f}}{k_{b}} \cdot \frac{C_{P}}{C_{S}}$ \\
\hline $\begin{array}{l}\text { Ordered mechanism } \\
\quad \text { (Inversed })^{*} \\
\text { OM(I) equation }\end{array}$ & 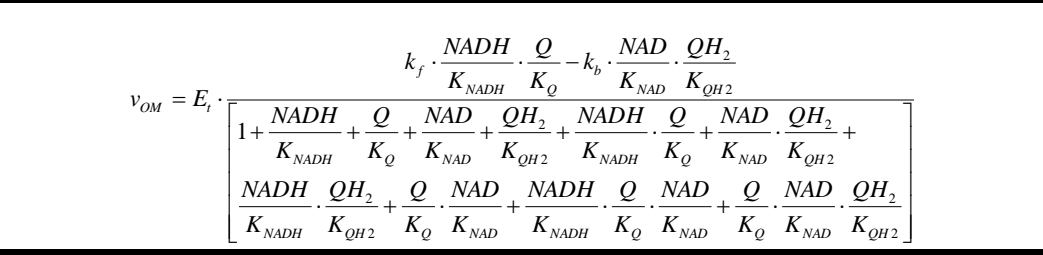 & $K_{e q}=\frac{k_{f}}{k_{b}} \cdot \frac{K_{N A D}}{K_{N A D H}} \cdot \frac{K_{Q H_{2}}}{K_{Q}}$ \\
\hline $\begin{array}{l}\text { Ping-Pong Mechanism } \\
\text { PPM equation }\end{array}$ & $v_{P P M}=E_{t} \cdot \frac{k_{f} \cdot \frac{N A D H}{K_{N A D H}} \cdot \frac{Q}{K_{Q}}-k_{b} \cdot \frac{N A D}{K_{N A D}} \cdot \frac{Q H_{2}}{K_{Q H 2}}}{\left[\begin{array}{l}\frac{N A D H}{K_{N A D H}}+\frac{Q}{K_{Q}}+\frac{N A D}{K_{N A D}}+\frac{Q H_{2}}{K_{Q H 2}}+\frac{N A D H}{K_{N A D H}} \cdot \frac{Q}{K_{Q}}+ \\
\frac{N A D}{K_{N A D}} \cdot \frac{Q H_{2}}{K_{Q H 2}}+\frac{N A D H}{K_{N A D H}} \cdot \frac{N A D}{K_{N A D}}+\frac{Q}{K_{Q}} \cdot \frac{Q H_{2}}{K_{Q H 2}}\end{array}\right]}$ & $K_{e q}=\frac{k_{\text {catf }}}{k_{\text {catb }}} \cdot \frac{K_{N A D^{+}} \cdot K_{Q H_{2}}}{K_{N A D H} \cdot K_{Q}}$ \\
\hline
\end{tabular}



Figure S2 : CK (red) vs ER-HMM (blue dashed). The experimental points are the same as in [28]. The curves e and $h$ are taken from curves $i$ and $j$ respectively with the NADH scale $0-30 \mu \mathrm{M}$.
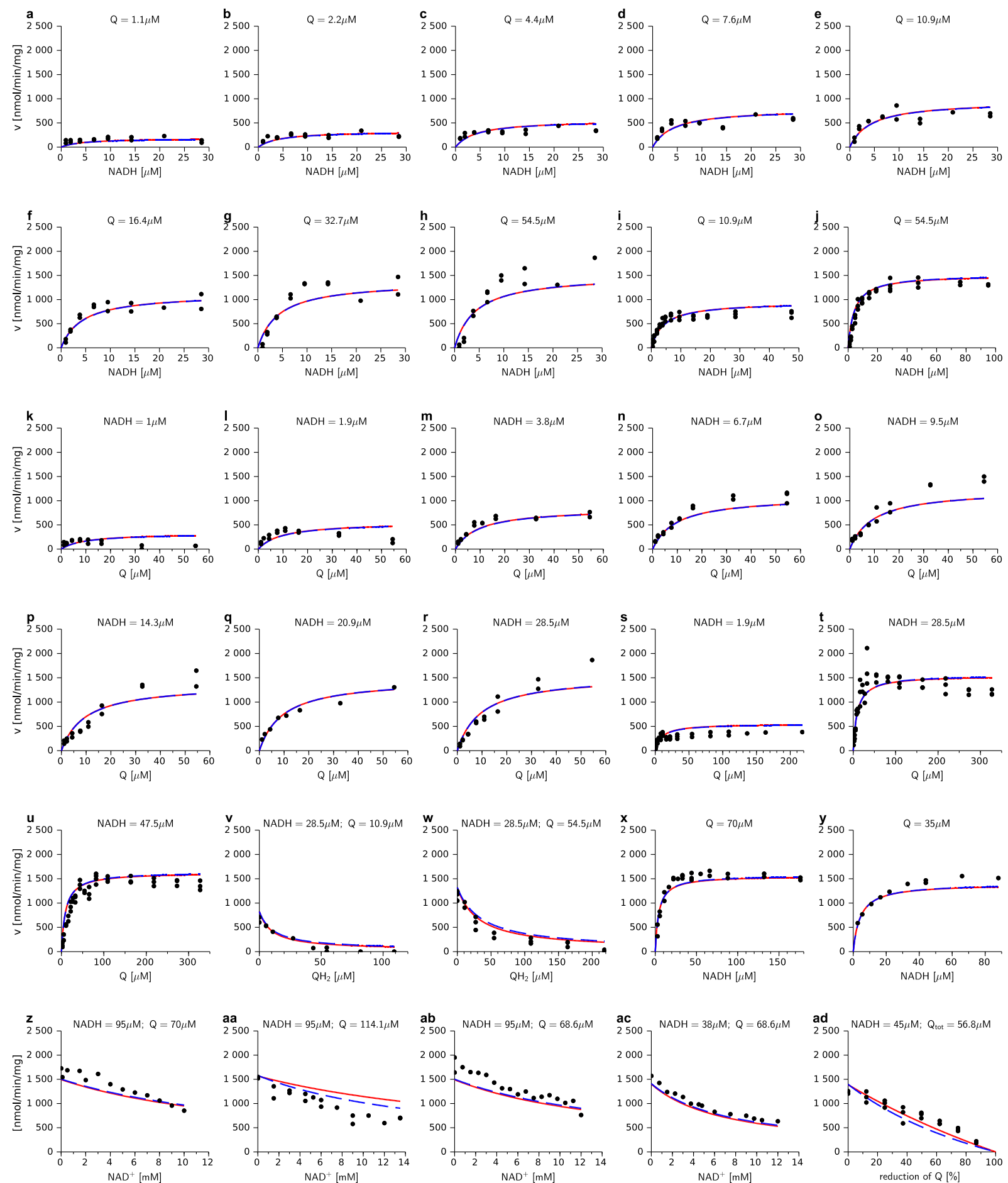
Figure S3: EMA (red) vs ER-HMM (blue dashed). The experimental points are the same as in [28]. The curves e and $h$ are taken from curves $i$ and $j$ respectively with the NADH scale $0-30 \mu \mathrm{M}$.
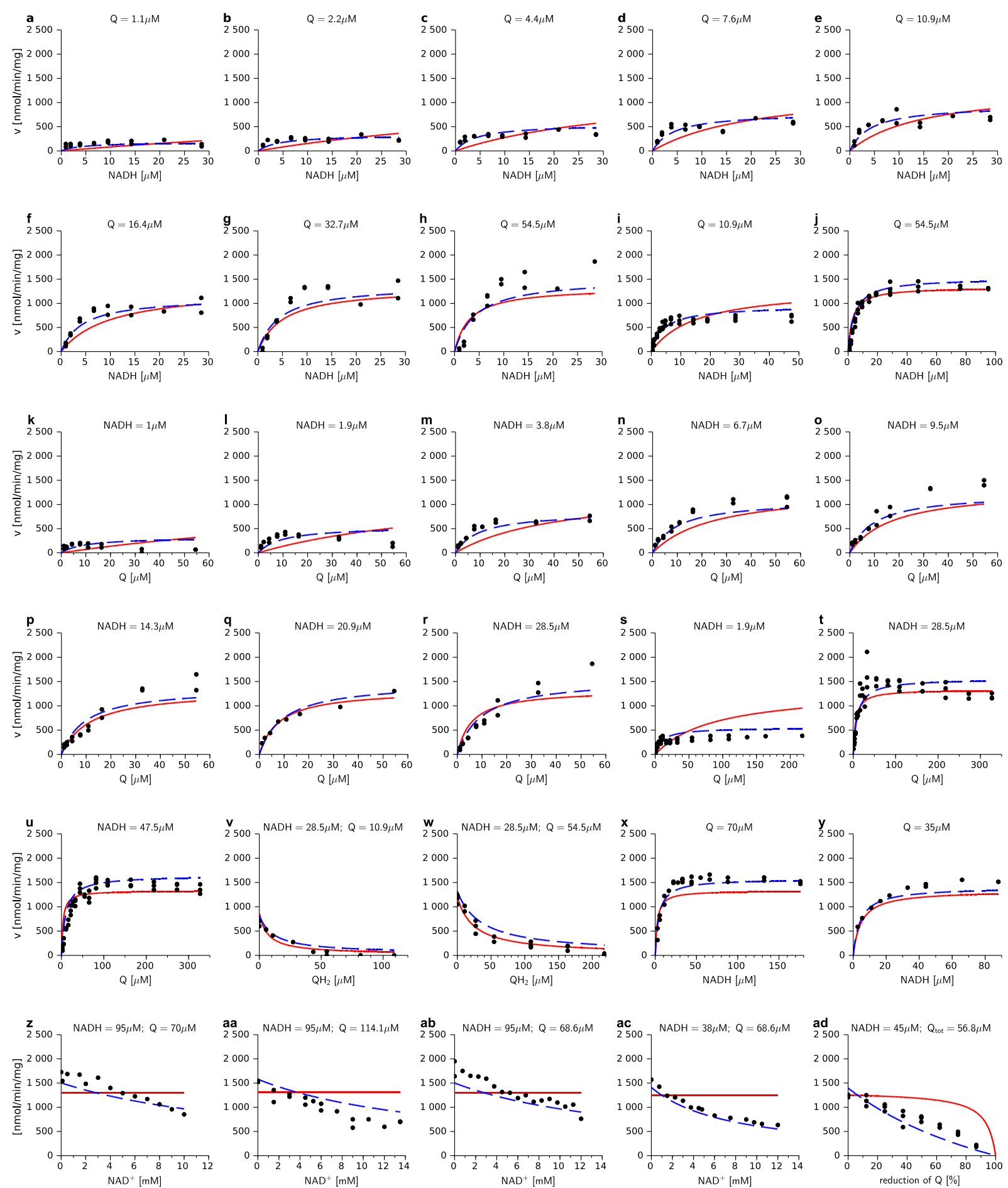
Figure S4: OM (NQNQ) (red) vs ER-HMM (blue dashed). The experimental points are the same as in [28]. The curves e and $h$ are taken from curves $i$ and $j$ respectively with NADH scale $0-30 \mu \mathrm{M}$.
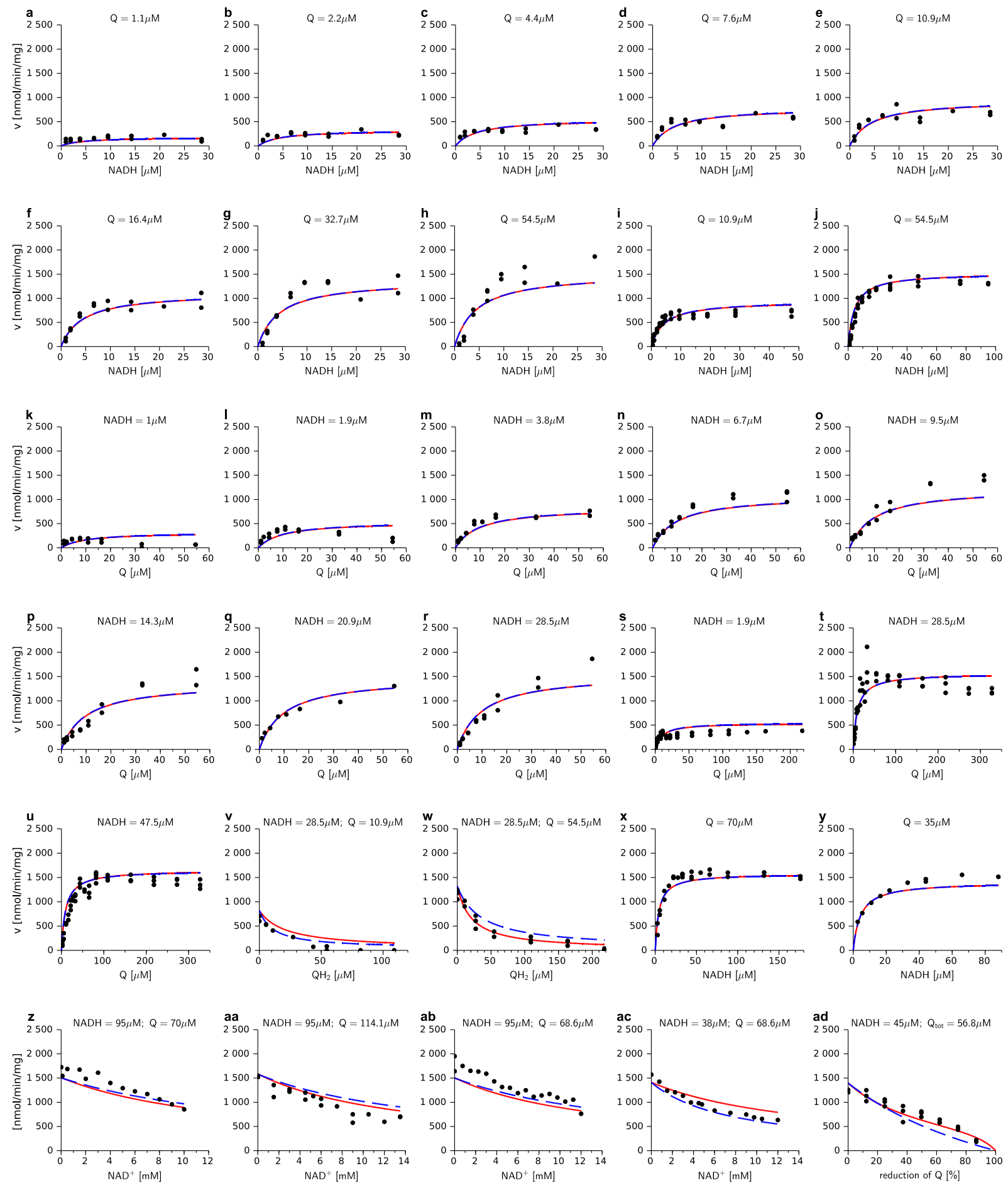
Figure S5: PPM (red) vs ER-HMM (blue dashed). The experimental points are the same as in [28]. The curves e and $h$ are taken from curves $i$ and $j$ respectively with NADH scale $0-30 \mu \mathrm{M}$.
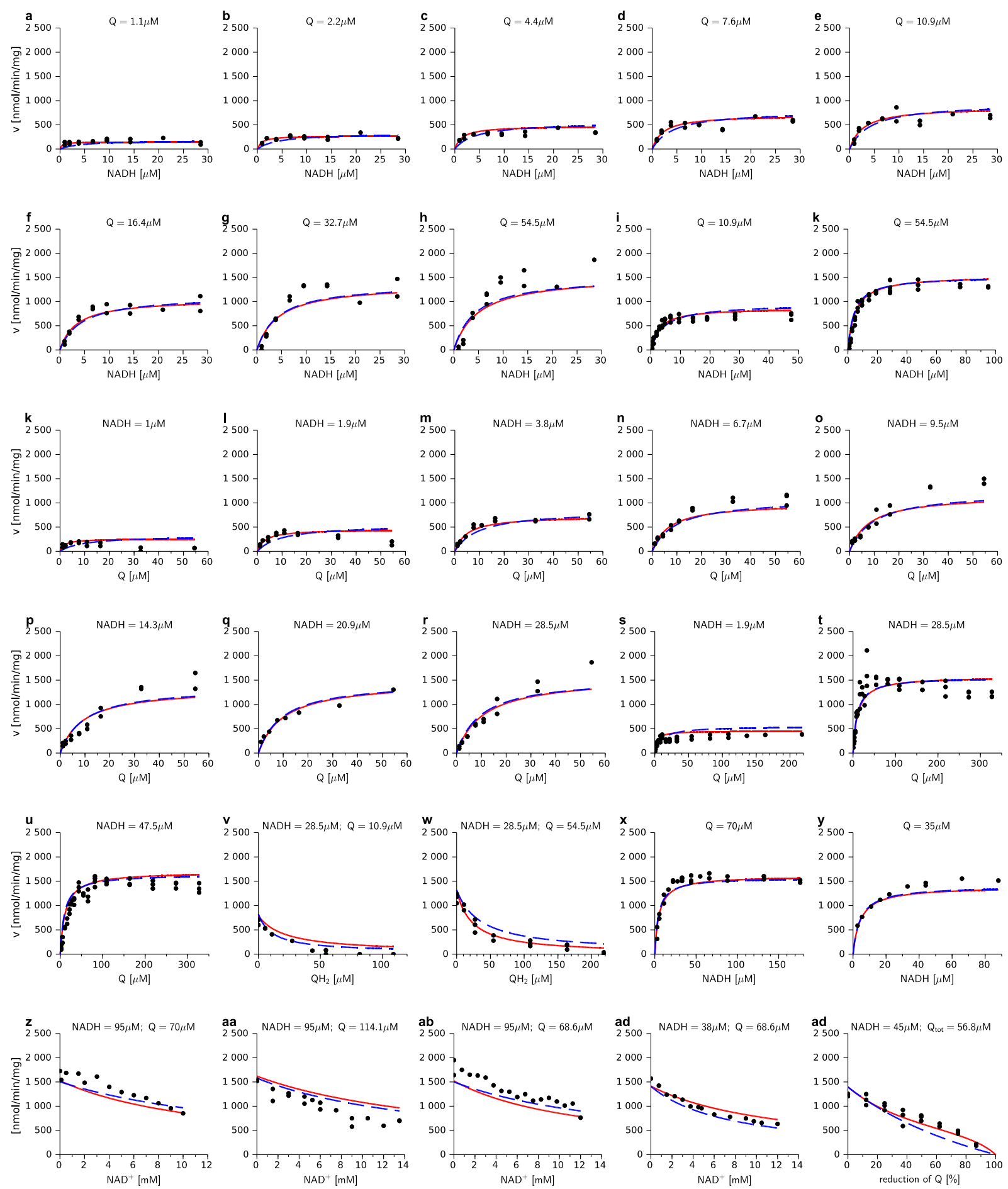
Figure S6: ER-HMM with (red) or without (blue dashed) non competitive inhibition by Q total. The experimental points are the same as in [28]. The curves e and $\mathrm{h}$ are taken from curves $\mathrm{i}$ and $\mathrm{j}$ respectively with NADH scale $0-30 \mu \mathrm{M}$.
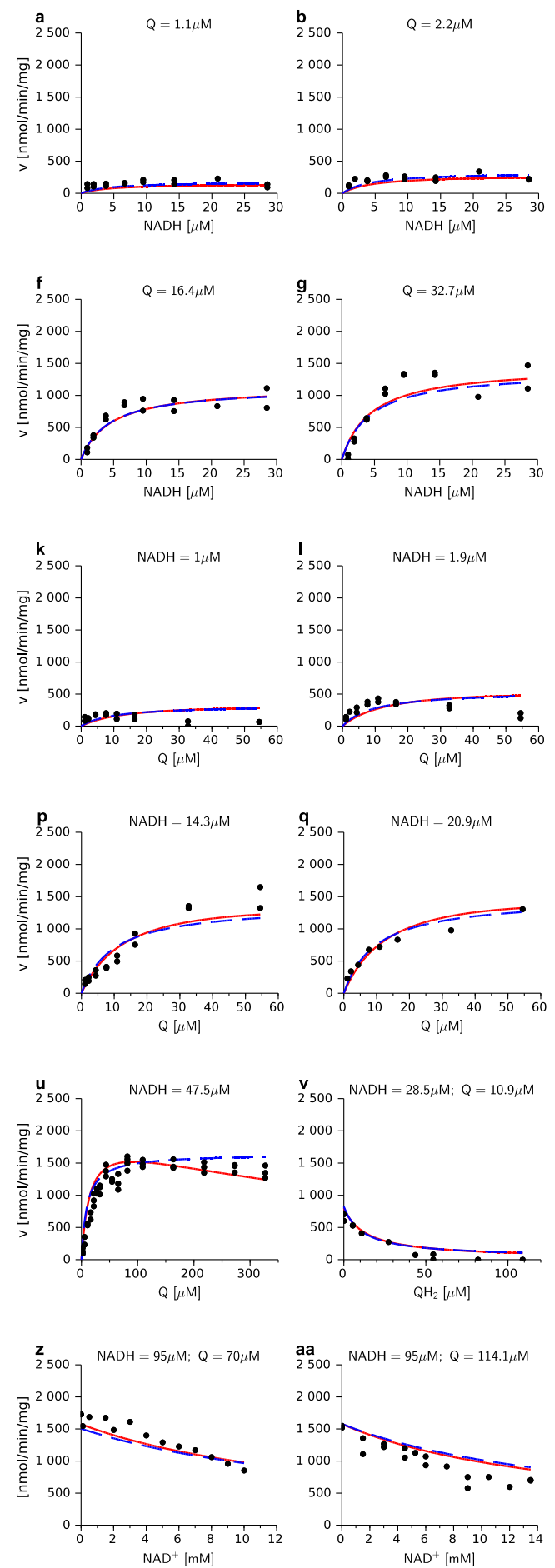
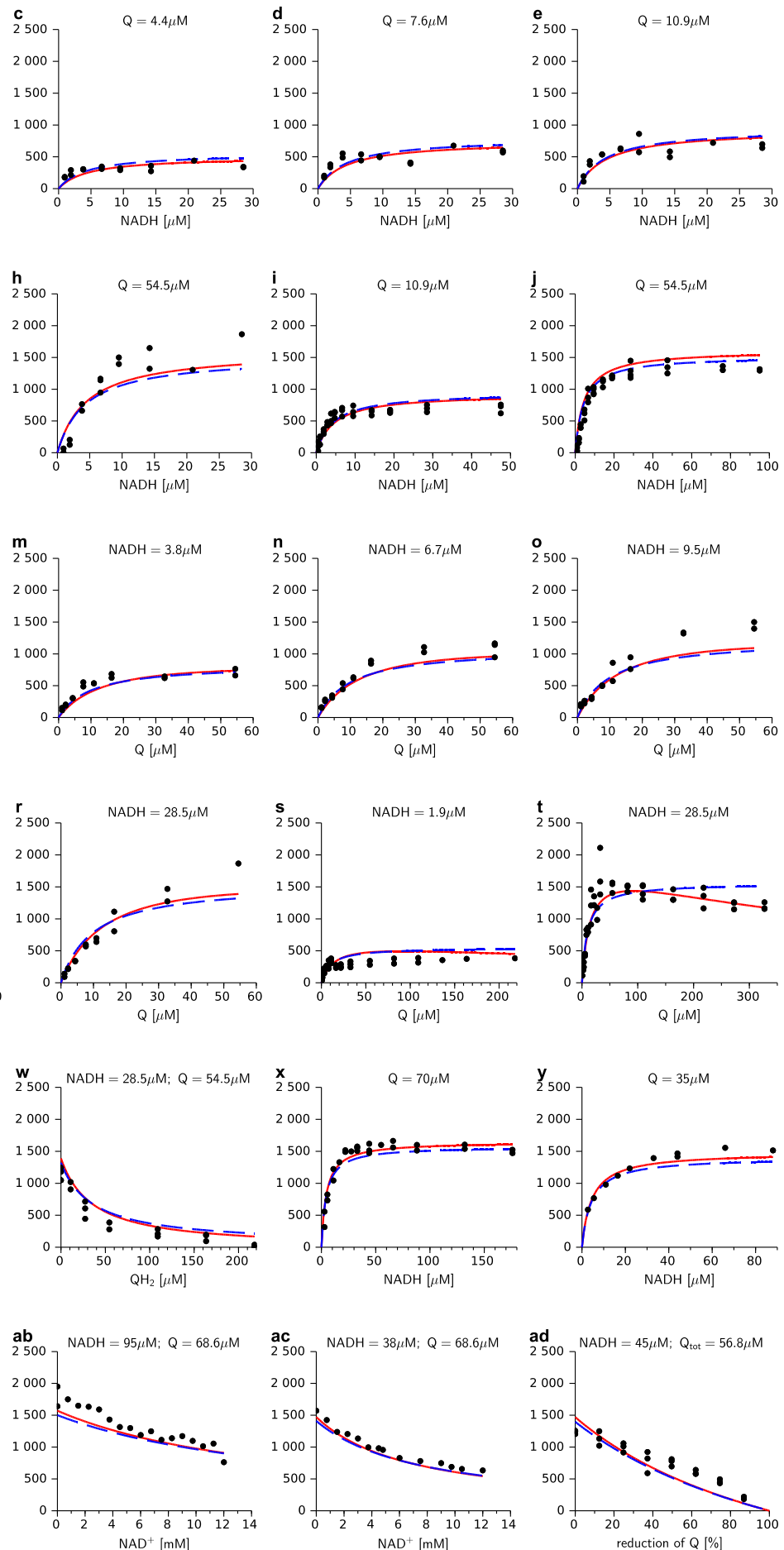
Figure S7: ER-HMM with (red) or without (blue dashed) steric inhibition by Q total. The experimental points are the same as in [28]. The curves e and $\mathrm{h}$ are taken from curves $\mathrm{i}$ and $\mathrm{j}$ respectively with the NADH scale $0-30 \mu \mathrm{M}$.
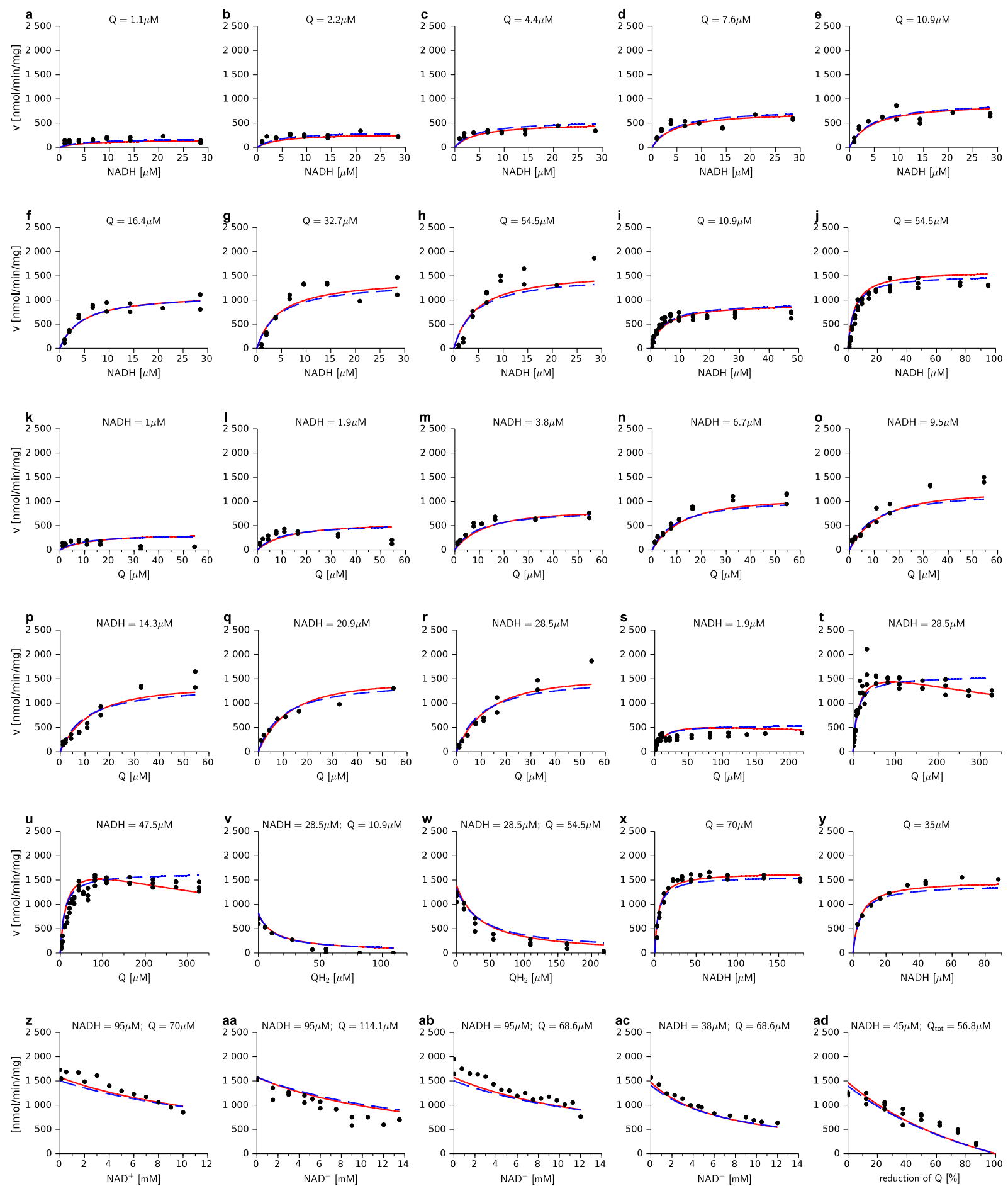


\section{SUPPLEMENTARY MATERIALS S8}

\section{Derivation of the extended mass action (EMA) equation: two possible mechanisms.}

The general form of the EMA equation is

$$
v=\left[E_{t}\right] \cdot \frac{k_{f} \cdot \frac{\prod_{i=1}^{m}\left[S_{i}\right]^{v_{i}}}{C_{S}}-k_{b} \cdot \frac{\prod_{j=1}^{n}\left[P_{j}\right]^{\eta_{j}}}{C_{P}}}{1+\frac{\prod_{i=1}^{m}\left[S_{i}\right]^{v_{i}}}{C_{S}}+\frac{\prod_{j=1}^{n}\left[P_{j}\right]^{\eta_{j}}}{C_{P}}}
$$

and is based on the net reaction

$$
\sum_{i=1}^{m} v_{i} \cdot S_{i} \Leftrightarrow \sum_{j=1}^{n} \eta_{j} \cdot P_{j} .
$$

It can be seen as a simplified ER-HMM equation in which the Michaelis constants of the substrates (resp. the products) are fused in one constant CS (resp. CP).

Theoretically, the number of the substrates $S$ and the products $\mathrm{P}$ ( $\mathrm{m}$ and $\mathrm{n}$, respectively) are not limited, and their stoichiometric factors ( $v$ and $\eta$, respectively) can take any positive value, also non integers.

The EMA equation (eq. SM 1) can be based on two different reaction mechanisms, both consisting of two steps-expressed in terms of mass action law. The first mechanism is close to the one used to derive enzyme rate laws. The second is based on the assumptions that the enzyme switches between two conformations during the reaction process.

\section{1) EMA equation - complex formation}

Following the early derivations of the Henri-Michaelis-Menten equation (Henri [31], Michaelis und Menten [32] and Briggs and Haldane, [33]. See also [34]), we introduce in the reaction mechanism the substrates-products-enzymes complexes species. We symbolize these species by only one intermediate state of the enzyme $\mathrm{E}^{*}$ which leads to the mechanism:

$$
E+\sum_{i=1}^{m} v_{i} \cdot S_{i} \stackrel{k_{1}}{\longleftarrow} E^{*} \underset{k_{-1}}{\stackrel{k_{2}}{\longleftrightarrow}} E+\sum_{j=1}^{n} \eta_{j} \cdot P_{j}
$$

where $k_{1}, k_{1}, k_{2}$ and $k_{2}$ are the forward and backward rate constants of these two reactions. Describing the two successive reactions in terms of mass action law leads to

$$
v_{1}=k_{1} \cdot[E] \cdot \prod_{i=1}^{m}\left[S_{i}\right]^{v_{i}}-k_{-1} \cdot[E *]
$$


$v_{2}=k_{2} \cdot[E *]-k_{-2} \cdot[E] \cdot \prod_{j=1}^{n}\left[P_{j}\right]^{h_{j}}$

Combining the equations SM 2 and SM 3 with the conservation of enzyme molecules, $\left[E_{t}\right]=[E]+\left[E^{*}\right]$, and with the classical assumption of steady-state, i.e. $v_{1}=v_{2}$ and $\frac{d\left[E^{*}\right]}{d t}=0$ we derive the rate equation

$$
v=\left[E_{t}\right] \cdot \frac{k_{2} \cdot \frac{k_{1}}{k_{2}+k_{-1}} \cdot \prod_{i=1}^{m}\left[S_{i}\right]^{v_{i}}-k_{-1} \cdot \frac{k_{-2}}{k_{2}+k_{-1}} \cdot \prod_{j=1}^{n}\left[P_{j}\right]^{\eta_{j}}}{1+\frac{k_{1}}{k_{2}+k_{-1}} \cdot \prod_{i=1}^{m}\left[S_{i}\right]^{v_{i}}+\frac{k_{-2}}{k_{2}+k_{-1}} \cdot \prod_{j=1}^{n}\left[P_{j}\right]^{\eta_{j}}}
$$

Setting $k_{f}=k_{2}$ and $k_{b}=k_{-1}, C_{S}=\frac{k_{2}+k_{-1}}{k_{1}}$ and $C_{P}=\frac{k_{2}+k_{-1}}{k_{-2}}$ we obtain the EMA equation (eq. SM 1).

\section{2) EMA equation - enzyme relaxation}

It has often been supposed that enzymes undergo conformational changes between different conformations with different activities [Frieden, 1979, Ricard, 1977 and 1979]. We assume here that the enzyme can adopt two conformations $E_{A}$ and $E_{B}$ in equilibrium. In addition we suppose that the substrates can only bind to the conformation $E_{A}$ and the products only to $E_{B}$. This implies, in order to the reaction to proceed, that there is an equilibrium reaction between $E_{A}$ and $E_{B}$. This is summarized by the two reactions below.

$E_{A}+\sum_{i=1}^{m} v_{i} \cdot S_{i} \stackrel{k_{1}}{\longleftrightarrow} E_{B}+\sum_{j=1}^{n} \eta_{j} \cdot P_{j}$

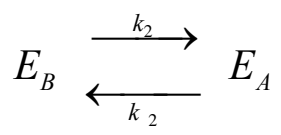

$k_{1}$ and $k_{1}$ are the forward and backward rate constants for the enzymatic step and $k_{2}$ and $k_{2}$ are the corresponding constants for the enzyme transconformational step. Describing the two steps in terms of mass action leads to

$$
v_{1}=k_{1} \cdot\left[E_{A}\right] \cdot \prod_{i=1}^{m}\left[S_{i}\right]^{r i}-k_{-1}\left[E_{B}\right] \cdot \prod_{j=1}^{n}\left[P_{j}\right]^{\eta_{j}}
$$

and 
$v_{2}=k_{2} \cdot\left[E_{B}\right]-k_{-2} \cdot\left[E_{A}\right]$.

Again, combining equations SM 5 and SM 6 with the conservation of enzyme molecules, $\left[E_{t}\right]=\left[E_{A}\right]+\left[E_{B}\right]$, and with the classical assumption of steady-state, i.e. $v_{1}=v_{2}$ and $\frac{d\left[E_{A}\right]}{d t}=\frac{d\left[E_{B}\right]}{d t}=0$, we derive the following equation :

$v=\left[E_{t}\right] \cdot \frac{k_{2} \cdot \frac{k_{1}}{k_{2}+k_{-2}} \prod_{i=1}^{m}\left[S_{i}\right]^{v_{i}}-k_{-2} \cdot \frac{k_{-1}}{k_{2}+k_{-2}} \prod_{j=1}^{n}\left[P_{j}\right]^{h_{j}}}{1+\frac{k_{1}}{k_{2}+k_{-2}} \prod_{i=1}^{m}\left[S_{i}\right]^{v_{i}}-\frac{k_{-1}}{k_{2}+k_{-2}} \prod_{j=1}^{n}\left[P_{j}\right]^{\eta_{j}}}$

By setting $k_{f}=k_{2}, k_{b}=k_{-2}, C_{S}=\frac{k_{2}+k_{-2}}{k_{1}}$ and $C_{P}=\frac{k_{2}+k_{-2}}{k_{-1}}$ we obtain again the EMA equation (equ. SM 1).

It must be pointed out that the same equation is obtained with two very different underlying mechanisms. When only one substrate and one product is present, the EMA equation (as well as the other equations cited in this paper - except for mass action law), simplify to the reversible Henri-Michaelis-Menten (HMM) equation.

This work was part of the "Diplomarbeit" of Margit Heiske, Bordeaux and Berlin June 2008. It was independently published with a different approach by Liebermeister et al. (2010) [35].

Bibliography.

J. Buc, J. Ricard, , J.-C. Meunier, Enzyme memory. 2. Kinetics and thermodynamics of the slow conformation changes of wheat-germ hexokinase LI.. Eur J Biochem. 80 (1977) 593-601.

C. Frieden, Slow transitions and hysteric behavior in enzymes. Annu Rev Biochem, 48 (1979) 471-489.

W. Liebermeister, J. Uhlendorf, E. Klipp, Modular rate laws for enzymatic reactions: thermodynamics, elasticities and implementation Bioinformatics. 26 (2010) 1528-34.

J.-C. Meunier, J. Buc, J. Ricard, Enzyme memory. Effect of glucose 6-phosphate and temperature on the molecular transition of wheat-germ hexokinase LI. Eur J Biochem. 97 (1979) 573-83.

J. Ricard, J. Buc, J.-C. Meunier, Enzyme Memory 1. A Transient Kinetic Study of Wheat-Germ Hexokinase LI. Eur J Biochem. 80 (1977) 581-592. 